\title{
A proteogenomic resource enabling integrated analysis of Listeria genotype-proteotype-phenotype relationships
}

\author{
Adithi R. Varadarajan ${ }^{1,2,4,9}$, Maria P. Pavlou ${ }^{1,3,4}$, ๆ, Sandra Goetze ${ }^{1,3,4}$, Virginie Grosboillot ${ }^{1,5}$, \\ Yang Shen ${ }^{1,5}$, Martin J. Loessner ${ }^{1,5}$, Christian H. Ahrens ${ }^{2, \&,}{ }^{*}$, Bernd Wollscheid $^{1,3,4, \&, *}$
}

1. Department of Health Sciences and Technology (D-HEST), ETH Zurich, 8092 Zurich, Switzerland

2. Agroscope, Molecular Diagnostics, Genomics \& Bioinformatics and SIB Swiss Institute of Bioinformatics, 8820 Wädenswil, Switzerland

3. BioMedical Proteomics Platform (BMPP), D-HEST, ETH Zurich, 8093 Zurich, Switzerland

4. Institute of Molecular Systems Biology (IMSB), ETH Zurich, 8093 Zurich, Switzerland

5. Institute of Food, Nutrition and Health (IFNH), ETH Zurich, 8092 Zurich, Switzerland

* Corresponding authors

Email: christian.ahrens@agroscope.admin.ch (CHA), wbernd@ethz.ch (BW)

१ These authors contributed equally to this work.

\& These authors contributed equally to this work.

Key words: Listeria, proteotype, proteogenomics, smORFs, DIA, PRM, EGD-e, ScottA 


\section{Abstract}

Listeria monocytogenes is an opportunistic foodborne pathogen responsible for listeriosis, a potentially fatal foodborne disease. Many different Listeria strains and serotypes exist, but a proteogenomic resource that bridges the gap in our molecular understanding of the relationships between the Listeria genotypes and phenotypes via proteotypes is still missing. Here we devised a next-generation proteogenomics strategy that enables the community to rapidly proteotype Listeria strains and relate this information back to the genotype. Based on sequencing and de novo assembly of the two most commonly used Listeria model strains, EGD-e and ScottA, we established two comprehensive Listeria proteogenomic databases. A genome comparison established core- and strain-specific genes potentially responsible for virulence differences. Next, we established a DIA/SWATH-based proteotyping strategy, including a new and robust sample preparation workflow, that enables the reproducible, sensitive, and relative quantitative measurement of Listeria proteotypes. This reusable and publically available DIA/SWATH library covers $70 \%$ of open reading frames of Listeria and represents the most extensive spectral library for Listeria proteotype analysis to date. We used these two new resources to investigate the Listeria proteotype in states mimicking the upper gastrointestinal passage. Exposure of Listeria to bile salts at $37{ }^{\circ} \mathrm{C}$, which simulates conditions encountered in the duodenum, showed significant proteotype perturbations including an increase of FlaA, the structural protein of flagella. Given that Listeria is known to lose its flagella above $30^{\circ} \mathrm{C}$, this was an unexpected finding. The formation of flagella, which might have implications on infectivity, was validated by parallel reaction monitoring and light and scanning electron microscopy. flaA transcript levels were not significantly different with and without exposure to bile salts at $37^{\circ} \mathrm{C}$, suggesting regulation at the post-transcriptional level. Together, these analyses provide a comprehensive proteogenomic resource and toolbox for the Listeria community enabling the analysis of Listeria genotype-proteotype-phenotype relationships. 


\section{Introduction}

Listeria monocytogenes is a highly adaptable environmental bacterium that can exist both as plant saprophyte and as animal pathogen [1]. The Gram-positive, rod-shaped, facultative anaerobic bacterium is the causative agent of listeriosis [2,3]. Although the incidence of listeriosis is relatively low compared to other common foodborne diseases, it is associated with one of the highest mortality rates (20\% - 30\%) [4]. Listeria strains are categorized into at least 14 serotypes [5], among these three $(1 / 2 a, 1 / 2 b$, and $4 b)$ are responsible for the majority of clinical cases. EGDe, a widely used model system of serotype $1 / 2 a$, is the serotype most frequently recovered from foods or food-processing plants. In contrast, ScottA is a widely used model system of serotype $4 b$, which causes the majority of human epidemics [5]. Infection by L. monocytogenes usually occurs after digestion of contaminated foods and in individuals with impaired cell-mediated immunity. The elderly, immunosuppressed patients, pregnant women, and neonates are particularly susceptible [2]. An infection may lead to meningitis, sepsis, or, by crossing the placenta, infection of the fetus and subsequent abortion [5]. Upon ingestion, L. monocytogenes must resist multiple stresses encountered in the gastrointestinal (GI) tract, including variation in $\mathrm{pH}$, osmolarity, and bile salts [6,7]. Notably, survival in the GI tract is a prerequisite to establish a successful infection in the host [8].

L. monocytogenes has served as a key bacterial model system to study host pathogen interaction [9], and studies of L. monocytogenes have led to the discovery of several new concepts in biology [10]. These included the discovery of unconventional mechanisms regulating bacterial gene expression, including the first RNA thermosensor regulating virulence [11], the excludon concept [12], and the discovery of an atypical member of the CRISPR family devoid of cas genes [10]. Additionally, research on L. monocytogenes has contributed to a better understanding of the structure and dynamics of the host cell cytoskeleton with the discovery of the first actin nucleator in eukaryotic cells (the Arp2/3 complex) $[13,14]$ and the elucidation of a novel role for clathrin in actin polymerization [10]. Finally, analysis of L. monocytogenes has been instrumental in characterizing naïve-to-memory CD8 T cell generation and differentiation [15]. 
The number of 'omics datasets that study different aspects of L. monocytogenes biology has increased exponentially in recent years. These datasets include a growing number of Illuminabased fragmented genomes [16] as well as complete genome sequences [17], the latter of which provide an optimal basis for comparative genomics and functional genomics studies. Such comparisons have helped to identify Listeria virulence factors and regions associated with pathogenicity $[18,19]$. Moreover, analyses of complete genomes enabled detailed investigations of genes transcribed under clinically relevant conditions [12] and enabled identification of novel protein coding genes and the correct protein $\mathrm{N}$-termini/start sites through proteogenomics [20]. Yet, changes in transcript abundances upon perturbation often do not correlate with abundance changes of the corresponding protein products [21,22], and transcriptional data alone does not provide important functional information about post-transcriptional regulation such as protein modifications [23] or changes in protein interaction networks or cell-surface remodeling of the host following an infection [24]. Such information can, however, be obtained using state-of-theart proteotype profiling.

The proteotype is defined as the state of the proteome at a particular time [25]. A proteome therefore consists of many proteotypes. The proteotype concept takes the dynamic nature of the proteome into account and extends it to the organization of proteins and their coexisting proteoforms in time and space [25]. Mass spectrometry-based proteotype profiling has matured recently through technological and methodological advances allowing for increased depth of proteome coverage [26-29] and sample throughput. This launches the next generation (next-gen) proteomics era of comprehensive and quantitative proteotype profiling. Recognizing the unmet need to integrate these datasets and to enable meta-analysis in a user-friendly manner, pioneers in the Listeria field developed the interactive Listeriomics website (https://listeriomics.pasteur.fr). At the time of publication, it contained 83 Listeria genome, 492 transcriptome, and 74 proteome datasets [30]. Notably, the majority of proteomic studies (qualitative and quantitative) were based on 2D gel studies, so modest numbers of proteins have been quantified thus far [31-37]. Similarly, the workflows employed to date have not provided the depth required for quantitative systems-level characterizations at the protein level. 
In the present study, we set out to generate and validate two proteogenomic resources to enable analysis of genotype-proteotype-phenotype relationships in L. monocytogenes strains. By relying on the de novo assembled genomes of the model strains ScottA and EGD-e, we generated a mass spectrometry-based toolbox using next-gen DIA/SWATH workflows to enable the sensitive, repetitive, and quantitative interrogation of $L$. monocytogenes proteotypes. DIA/SWATH-MS (for Data Independent Acquisition/Sequential Window Acquisition of all THeoretical Mass Spectra) is a recently introduced proteotyping technology based on mass spectrometry, which, compared to data-dependent acquisition (DDA) MS strategies, enables more sensitive generation of comprehensive proteotype datasets. The dynamic range of DIA/SWATH-MS is in excess of four orders of magnitude [38], which matches the dynamic range of the proteotypes expected for our L. monocytogenes strains. Recently, proteogenomic studies on Streptococcus pyogenes [39] and Mycobacterium tuberculosis [40] utilizing the DIA/SWATH technology have illustrated its impact, which led to new biological insights with respect to invasiveness of clinical isolates and to dormancy and resuscitation, respectively.

We applied our newly developed proteogenomic resources and toolbox to investigate how L. monocytogenes cells cope with stress encountered during passage through the upper GI tract, a prerequisite for systemic infection of the host. We uncovered evidence for the unexpected expression of flagella upon exposure to bile salts at $37^{\circ} \mathrm{C}$, a condition mimicking the duodenum. The comprehensive proteogenomic resource and toolbox we established here will enable further analyses of the Listeria genotype-proteotype-phenotype relationships. 


\section{Results \& Discussion}

\section{A generic, genomics-driven strategy enabling the investigation of genotype- proteotype-phenotype relationships}

We selected two widely used L. monocytogenes strains, EGD-e and ScottA, as model systems to evaluate the applicability of our genomics-driven next-gen proteomics workflow (Fig 1). EGD-e and ScottA belong to serotypes $1 / 2 a$ and $4 b$, respectively, which are responsible for the majority of listeriosis cases (S1 Table); ScottA is more invasive than EGD-e [41]. We relied on an integrated workflow to obtain a quantitative profile of the Listeria proteotype. This workflow contains four main components: genomics, comparative genomics, proteotype analysis, and proteogenomics (Fig 1).

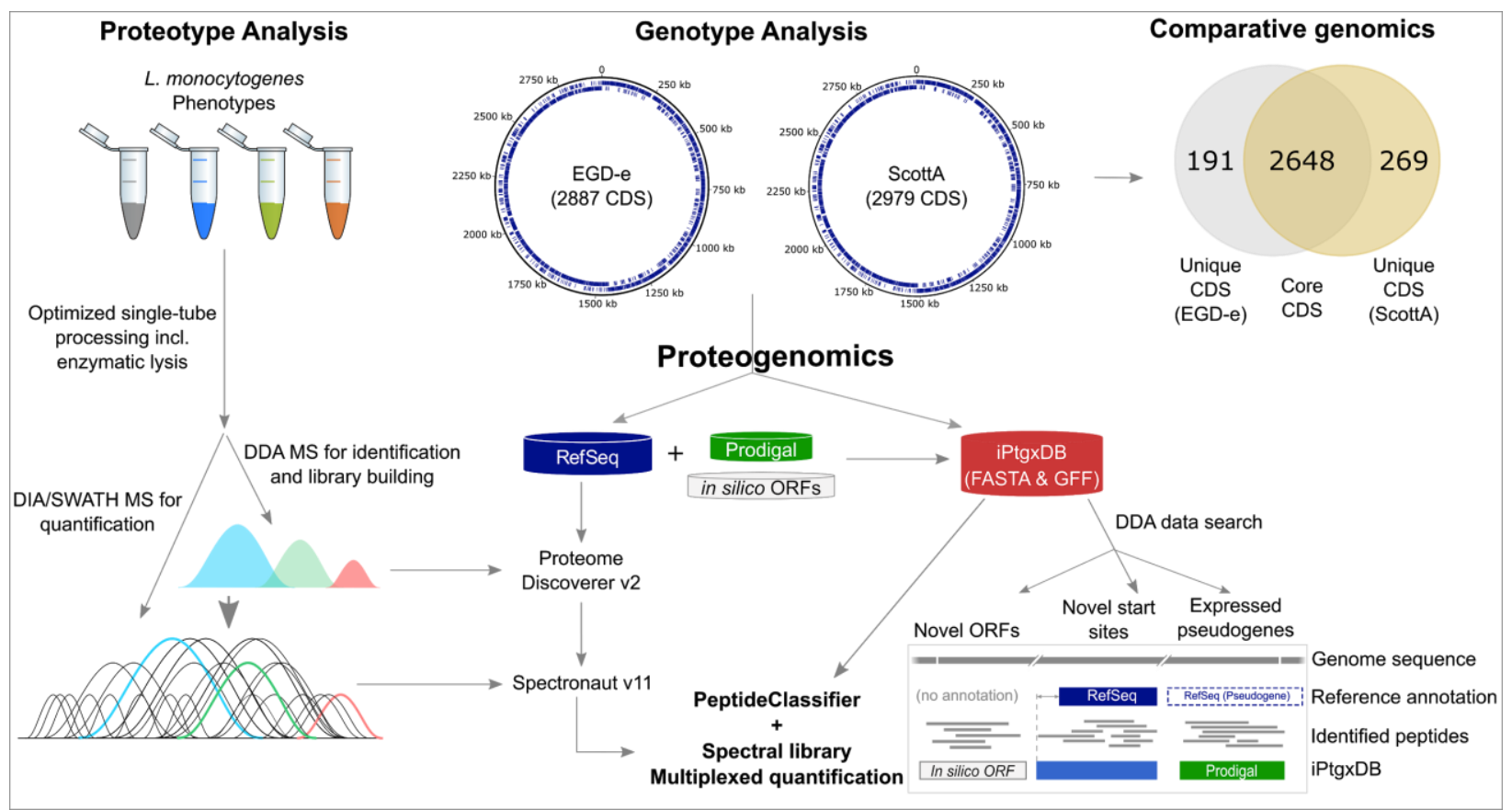

Fig 1. Overview of our next-gen proteogenomics workflow for $L$. monocytogenes. A sample preparation method was developed that allows rapid, reproducible single-tube reactions for lysis, digestion, desalting, and subsequent proteotype profiling with DDA- and DIA-MS (left panel). In parallel, the genomes of both EGD-e and ScottA were de novo assembled into complete, high-quality genome sequences, and their RefSeq annotations were obtained from the NCBI's prokaryotic genome annotation pipeline (PGAP) [42] 
(middle upper panel). Comparative genomics identified both shared core gene clusters and gene clusters unique to EGD-e and ScottA (right upper panel). Moreover, an ab initio gene prediction based on Prodigal and an advanced in silico (six-frame translation) annotation (see Materials and Methods) were integrated with the RefSeq annotation to obtain a minimally redundant iPtgxDB [43] for EGD-e and for ScottA. DDAbased proteomics data were searched against the publicly available iPtgxDBs (https://iptgxdb.expasy.org) to obtain proteogenomic evidence for novel open reading frames (ORFs), novel start sites, and expressed pseudogenes (right lower panel). For the proteotype analysis, proteomics data obtained from DDA mode were searched against the RefSeq annotations and spectral libraries were generated with Spectronaut. These publicly available resources were then used to analyze and quantify proteins obtained from DIA mode.

The first step in our workflow was a genomics analysis. Although a complete NCBI reference genome sequence existed for EGD-e, the NCBI reference genome sequence for ScottA was incomplete and consisted of five contigs [44]. Motivated by our recent finding of significant differences between an NCBI reference genome and the de novo assembly of the actual lab strain [43], and an earlier study on Pseudomonas aeruginosa PAO1 that had demonstrated substantial genomic fluidity between closely related strains [45], we sequenced and de novo assembled both genomes to create the best possible reference sequence for the two strains, an important aspect for the proteogenomics element. Next, a comparative genomics analysis was carried out to identify core and strain-specific genes, which, upon integration with protein abundance data, might provide clues to explain the different phenotypes of the strains. Thirdly, we performed DDA-MS experiments under relevant conditions to obtain extensive Listeria proteotype datasets, which became the basis for the construction of Listeria spectral libraries. After establishing a rapid and reproducible sample preparation workflow, we were able to quickly and quantitatively profile Listeria under various conditions and perturbations by DIA/SWATH. Lastly, in addition to the standard proteotype search against generic protein databases such as NCBI RefSeq and Uniprot, a search was carried out against a specialized, integrated proteogenomics search database (iPtgxDB; see Materials and Methods) [43], allowing us to identify protein expression evidence for as of yet unannotated small ORFs (smORFs), additional N-terminal start sites, and expressed pseudogenes (Fig 1). 
A variant of such a proteogenomics approach recently revealed $\mathrm{N}$-terminal peptides both from internal start sites of annotated L. monocytogenes EGD-e proteins and from six novel, unannotated smORFs including Prli42 [20]. This 31-amino acid protein relays oxidative stress signals to the stressosome to activate the general stress-sensing pathway, the sigma $\mathrm{B}$ regulon, and represents the long-sought link between stress and the stressosome [46]. Despite the many important functions of smORFs, such small, functional open reading frames are often missed in current genome annotations [47]. Proteogenomics and ribosome profiling have emerged as the most important technologies for comprehensive identification of smORFs [47]. Consequently, we added a proteogenomics element to our generic strategy. This has two benefits: First, proteogenomics can be included in the initial genome annotation, thereby increasing its quality $[43,48]$. A public website (https://iptgxdb.expasy.org/) supports this for newly sequenced genomes [43], such as isolates from microbiomes or type strains from the Genomic Encyclopedia of Bacteria and Archaea (GEBA) initiative, which aims to expand the phylogenetic diversity of completely sequenced prokaryotic genomes [49]. Second, the identification of more comprehensive protein catalogs including functionally relevant smORFs, will better enable studies to model systems based on quantitative data of all functional elements.

\section{Complete genome sequences of EGD-e and ScottA and comparative genomics}

An analysis of the repeat complexity of all publicly available, completely sequenced genomes of L. monocytogenes strains (status: March, 2018) revealed that almost 95\% of the roughly 150 strains are so-called "class I" genomes, which are straightforward to assemble (few repeats, none longer than the rDNA operons of up to $7 \mathrm{~kb}$ ) [17]. In contrast, eight strains also had few repeats overall, but those present were up to $11 \mathrm{~kb}$ in length [17]. Using long-read Pacific Biosciences (PacBio) sequencing data (including a BluePippin size selection step; see Materials and Methods) and the assembly algorithm HGAP3 [50], we were able to de novo assemble one complete chromosome for EGD-e (2.94 Mbp) and one for ScottA (3.03 Mbp) with PacBio read coverages of 260x and 280x, respectively (Fig 2). To correct remaining homopolymer errors and to remove small insertions or deletions (INDELs) in the PacBio data [51], both strains were also sequenced using the highly accurate short-read Illumina protocol. The Illumina data allowed us 
to ensure that no additional plasmids could be assembled that might have been lost in the BluePippin size selection step before creating the insert libraries for PacBio sequencing. Additional genome properties, including the number of protein-coding genes predicted by NCBI's PGAP annotation pipeline, are listed in S2 Table. Two intact and one putative prophage were identified by PHASTER [52] in ScottA, and two putative prophages were identified in EGD-e (Materials and Methods).
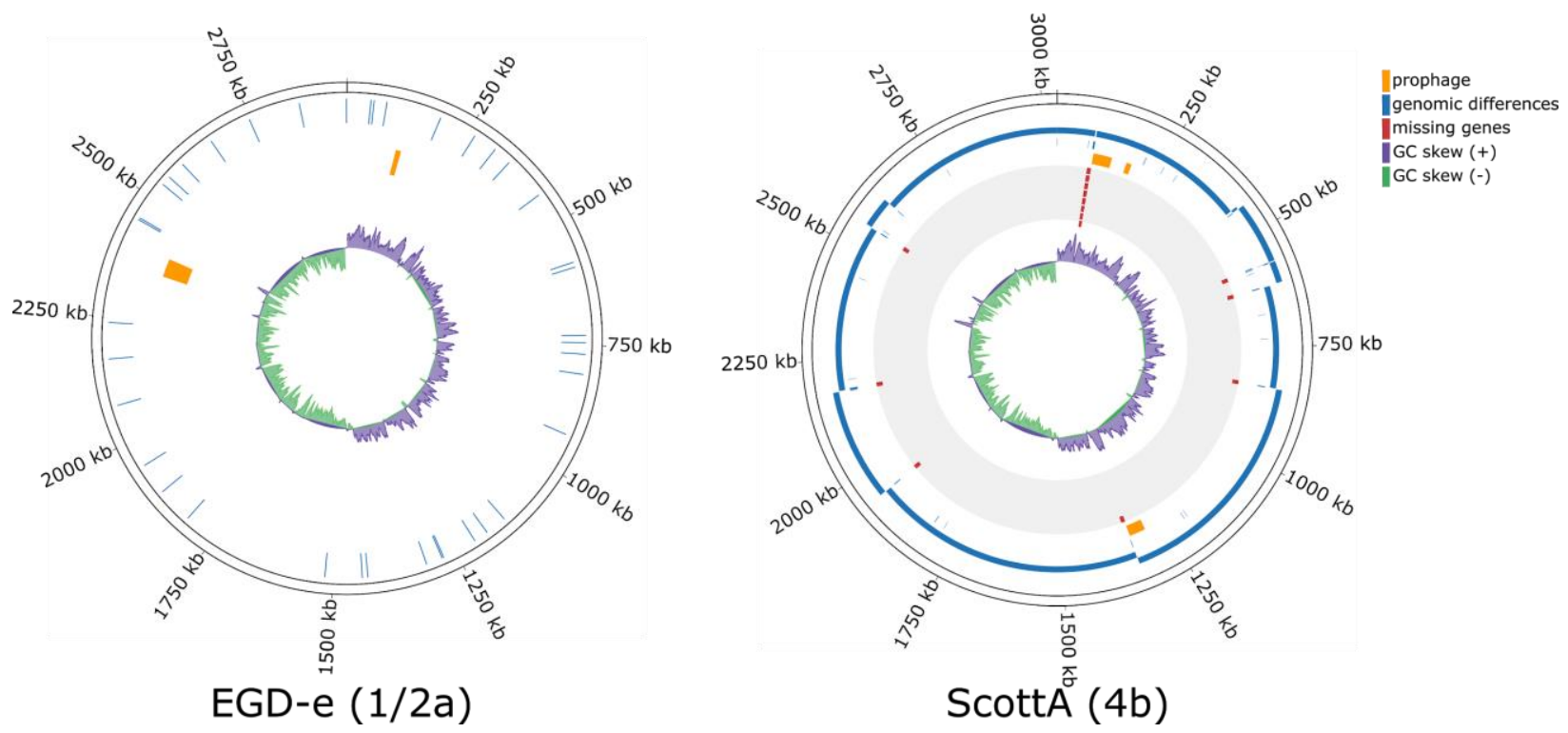

Fig 2. Circular plots showing the de novo-assembled genome sequences (outer ring with genomic coordinates) of EGD-e and ScottA and the genomic differences compared to the existing reference sequences, NC_003210 and NZ_CM001159, respectively. Whereas our EGD-e assembly exhibited minor differences compared to the NCBI reference genome (SNVs and INDELS in blue, second circle), there were a number of differences between our complete ScottA assembly and the NCBI reference (incomplete, 5 contigs), which had been assembled using a reference-based assembly approach [44]. The mapping of the five contigs and the remaining gaps (blue) are shown in the second circle; prophages (orange) in the third, 15 missing genes (red) in the fourth, and the GC skew (positive, purple; negative, green) in the fifth circle.

Comparing our de novo assembled ScottA genome to the NCBI reference (CM001159.1; 5 contigs), we observed a total of 11,953 base pairs (bp) of missing sequence, which affected 14 genes that were completely or partially missed in the earlier, at the time state of the art, 
reference-based genome assembly [44]. The missing genes included seven hypothetical proteins, three surface proteins, one cell-wall anchor-domain containing protein, and three transposases (S3 Table). The genomic differences included 14 insertions, 16 deletions, 93 single-nucleotide variations (SNVs), and 34 variations affecting two or more nucleotides (Fig 2). In contrast, only 28 SNVs and 11 single-bp INDELs were observed between our EGD-e assembly and the NCBI reference genome (NC_003210.1) (Fig 2).

The example of ScottA illustrates that a de novo assembly strategy is preferable over a reference-based one, which can easily miss important genome sequence differences. Roughly 570 of 9,331 bacterial genome assemblies (6.1\%) that we recently analyzed [17] were prepared using a reference-based genome assembly strategy; these assemblies thus have to be treated with caution. With current long-read sequencing technologies like Pacific Biosciences and Oxford Nanopore Technologies readily generating sequence reads longer than $20 \mathrm{~kb}$, de novo genome assembly has become the preferred approach.

Finally, complete genomes also represent the best basis for comparative genomics studies, as core genes have been missed when more fragmented assemblies based on short Illumina reads were used for comparative genomics [53]. A comparison of the complete genomes of EGD-e and ScottA using Roary [54] revealed a high similarity with 2,648 core gene clusters (orthologous proteins), 191 (6.6\%) EGD-e-specific and 269 (9\%) ScottA-specific gene clusters (Fig 1 and Table 1). Notably, five of the fourteen genes that were missed in the five contigs of the fragmented ScottA genome are indeed core genes that are shared by both strains. A list of core and strain-specific genes is provided in S3 Table. These results will enable the Listeria research community to further explore differing virulence capabilities and other properties of these two strains. To facilitate such comparisons and integration with other data sets, we also provide a detailed table with the genes of both strains, functional annotations, proteomic abundance evidence, and a reciprocal best BLAST hit comparison against the ListiList EGD-e proteins with identifiers in the form of LmoXXXX (https://listeriomics.pasteur.fr), where X is a number from 09) in S4 Table. Together, these new proteogenomic resources represent a high-quality set of puzzle pieces required for modeling and understanding the Listeria life cycle and infectious mode of action. 
Table 1. Overview of the comparative genomics results of the newly sequenced and assembled EGD-e and ScottA genomes including core and strain-specific protein-coding genes.

\begin{tabular}{|l|r|r|}
\hline & $\begin{array}{r}\text { L. monocytogenes } \\
\text { EGD-e }\end{array}$ & \multicolumn{2}{|c|}{$\begin{array}{c}\text { L. monocytogenes } \\
\text { ScottA }\end{array}$} \\
\hline Genbank accession \# & CP023861 & CP023862 \\
\hline Size of the chromosome (bp) & $2,944,523$ & $2,030,813$ \\
\hline Total number of protein-coding genes & 2,887 & 269 \\
\hline Number of strain-specific genes & 191 & 2,648 \\
\hline Number of core genes & 2,648 & 2,0 \\
\hline
\end{tabular}

Growth under conditions mimicking passage through the gastrointestinal tract

Despite intense research, knowledge of the proteotype adaptations that facilitate survival of $L$. monocytogenes in the GI tract remains incomplete. To cause a systemic infection, the bacteria have to be ingested and travel through the Gl tract [8]. The GI tract is a hostile environment, and the bacteria are subjected to mechanical and chemical stresses that differ depending on the compartment: These include acidity (stomach), exposure to bile (duodenum), and exposure to high osmolarity (jejunum) (Fig 3A). To enable a systems-wide, quantitative characterization of the proteins required for survival and adaptation of L. monocytogenes under these Gl-imposed stresses, we first sought to devise in vitro culturing conditions that resemble the different microenvironments of the Gl tract. As not all genes will be expressed under one condition, these perturbations were chosen in order to obtain a broad representation of expressed Listeria proteins. We reasoned that discovery-driven DDA-based proteotype data from such conditions would allow us to create a comprehensive spectral library, an important element of our next-gen DIA/SWATH-based proteotyping strategy (Fig 1). This was achieved by culturing L. monocytogenes cells in three different conditions at $37^{\circ} \mathrm{C}$ [55] and in a control condition (buffered peptone water (BPW), $\mathrm{pH}$ 7.4) in which the cells did not replicate (based on $\mathrm{OD}_{600}$ measurements, data not shown). 
A
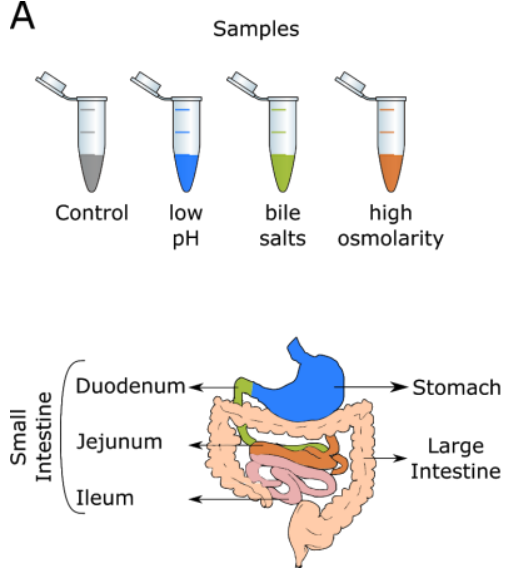

B
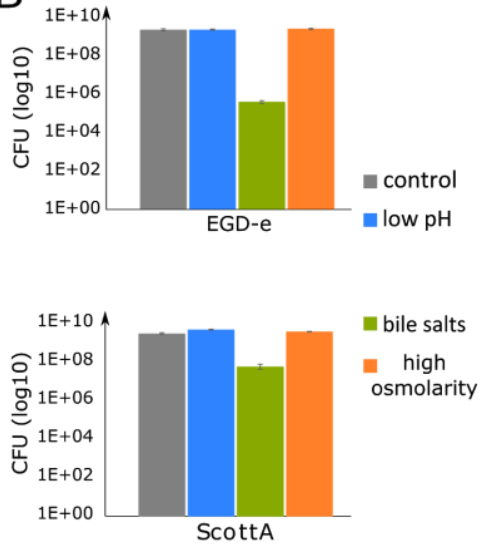

C

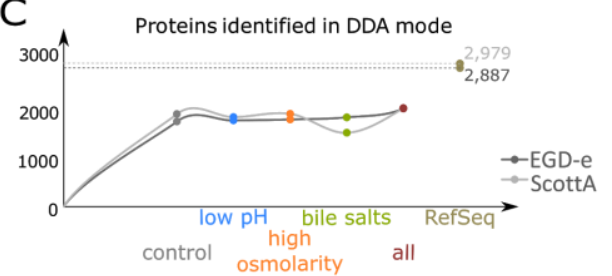

\begin{tabular}{|c|c|c|}
\hline & EGD-e & ScottA \\
\hline control & $1,771(61 \%)$ & $1,927(65 \%)$ \\
\hline low pH & $1,794(62 \%)$ & $1,865(63 \%)$ \\
\hline \hline high osmolarity & $1,815(63 \%)$ & $1,933(65 \%)$ \\
\hline bile salts & $1,860(64 \%)$ & $1,537(52 \%)$ \\
\hline all conditions & $2,042(71 \%)$ & $2,052(69 \%)$ \\
\hline RefSeq & 2,887 & 2,979 \\
\hline
\end{tabular}

Fig 3. Discovery-driven DDA-based Listeria protein expression data obtained under conditions mimicking passage through the upper GI tract. (A) Sample conditions (control, grey; stomach, blue; duodenum, green; jejunum, orange). (B) Viability of cells in control and three different conditions. (C) Number of proteins identified per condition for each strain, the overall number of identified proteins (red), and the number of proteins annotated in each strain (brown).

After testing several combinations (data not shown) the following buffer compositions were chosen (Fig 3A): To mimic conditions encountered in the stomach, the $\mathrm{pH}$ of the buffer was 4 , and the medium included 1,000 units $/ \mathrm{ml}$ of pepsin. To simulate the duodenum, the medium included $0.3 \% \mathrm{w} / \mathrm{v}$ bile salts ( $\mathrm{pH} 7.4$ ). Finally, in order to mimic conditions encountered in the jejunum, the $\mathrm{pH}$ of the medium was 8 , and it contained $0.3 \mathrm{M}$ sucrose to increase the osmolarity (Fig 3A). The viability of the strains was not affected by the stresses of low $\mathrm{pH} / \mathrm{pepsin}$ and high $\mathrm{pH} /$ high osmolarity (Fig 3B). In contrast, viability was severely compromised by the presence of bile salts, indicating that detergent-like activities are detrimental to survival of bacterial cells. In the presence of bile salts, the viability of ScottA (approximately one log lower than in the control) was affected much less than that of EGD-e (approximately 4 logs lower compared to the control) (Fig 3B). The greater sensitivity of EGD-e to both $\mathrm{pH}$ and bile compared to other L. monocytogenes strains, including ScottA, has been noted before $[6,7]$. Although these are clearly model conditions, in the absence of better in vivo models, they will lead to a better understanding of 
the mechanisms by which Listeria adapts to the host GI tract, informing the development of novel treatment or prophylactic strategies.

\section{Listeria proteotype analysis using a single-tube workflow and spectral libraries}

To ensure reproducible, sensitive, and quantitative proteotype measurements both for this study and as a general resource for the Listeria research community, a robust sample preparation workflow was needed that included a minimal number of sample preparation steps, while simultaneously enabling a comprehensive protein identification and quantification across conditions/proteotypes. Typically, bacterial cells are lysed mechanically (i.e., by bead beating) or by use of detergents. However, these methods require additional steps for bead or detergent removal making the workflows more tedious, increasing technical variability, and potentially leading to loss of low abundance proteins. Therefore, an efficient and proteotype analysiscompatible workflow was developed in which all steps from lysis to protein digestion are performed in a single tube (Fig 1). Effective, rapid, and complete cell lysis was achieved by incubating L. monocytogenes cells with the bacteriophage endolysin (Ply511; see Materials and Methods). Recombinantly produced endolysins applied exogenously to susceptible bacteria display the same lytic properties as their native counterparts [56]. In combination with indirect sonication, combined LysC/trypsin protein cleavage into peptides and desalting using a mixed cationic exchange resin, the sample processing strategy enables rapid proteotyping of $L$. monocytogenes.

To generate spectral libraries, samples were first analyzed in DDA-MS mode. For this, the raw files were analyzed with MaxQuant "dependent peptide" settings [57] to identify the most prominent post-translational modifications, which were then used as variable modifications in a second search, thereby limiting the overall search space. In total, we identified qualitative expression data for between 1,700 and 1,800 proteins per condition for EGD-e and between 1,500 and 1,900 proteins per condition for ScottA (Fig 3C), similar to previously reported, extensive MuDPIT datasets from Listeria [36,58]. We next used the discovery-driven DDA-based data to generate spectral libraries for DIA/SWATH-based acquisition and reliable protein 
quantification across conditions. Summaries of the spectral library characteristics for the two strains are depicted in Table 2 and Fig 4.

Table 2. The numbers of precursors, peptides, and proteins that are covered in the spectral libraries of $L$. monocytogenes strains EGD-e and ScottA and peptides that were observed for strain-specific proteins.

\begin{tabular}{|c|c|c|c|c|c|c|}
\hline & $\begin{array}{c}\text { Proteins } \\
\text { (strain-specific) }\end{array}$ & Precursors & Peptides & $\begin{array}{c}\text { Proteotypic } \\
\text { (per strain) }\end{array}$ & $\begin{array}{c}\text { Proteotypic } \\
\text { (between } \\
\text { strains) }\end{array}$ & $\begin{array}{c}\text { Proteotypic peptides of } \\
\text { strain-specific proteins }\end{array}$ \\
\hline EGD-e & $1,992(71)$ & 379,848 & 22,520 & $\begin{array}{c}22,520 \\
(100 \%)\end{array}$ & $3,493(15.5 \%)$ & $544(0.02 \%)$ \\
\hline ScottA & $2,002(71)$ & 479,018 & 22,768 & $\begin{array}{c}22,768 \\
(100 \%)\end{array}$ & $3,681(16.2 \%)$ & $591(0.03 \%)$ \\
\hline
\end{tabular}

Each library contained roughly 22,000 peptides that corresponded to approximately $2,000$ proteins (false discovery rate $(F D R)<1 \%)$ ). All of the peptides were proteotypic for the given strain as indicated by a PeptideClassifier analysis [59] when using the peptide and protein identifications returned from ProteomeDicoverer as input (see Materials and Methods). Overall, the spectral libraries contained roughly $69 \%$ and $67 \%$ of the annotated ORFs of EGD-e and ScottA, respectively. To our knowledge, this is the first report of Listeria-specific spectral libraries covering such a high proportion of the theoretical proteome. A Gene Ontology (GO) enrichment analysis of the proteins contained in the spectral libraries showed that the majority of pathways were represented in both of the libraries and that similar GO categories were enriched in both strains across the three domains. Notably, the cellular component (CC) 'cytoplasm' was significantly over-represented among proteins in the spectral libraries of both strains (Fig 4), whereas proteins associated with the CC category 'transmembrane' were under-represented (data not shown). This is an expected outcome and can be explained by our focus on the development of a single-tube, rapid and reproducible sample processing workflow. Proteome coverage without a bias against the membrane proteome has only very rarely been achieved and requires elaborate strategies (computational and biochemical and/or subcellular fractionation) and substantial effort [27]. The CC categories 'cytosol' and 'cell wall' were also over-represented. 
A
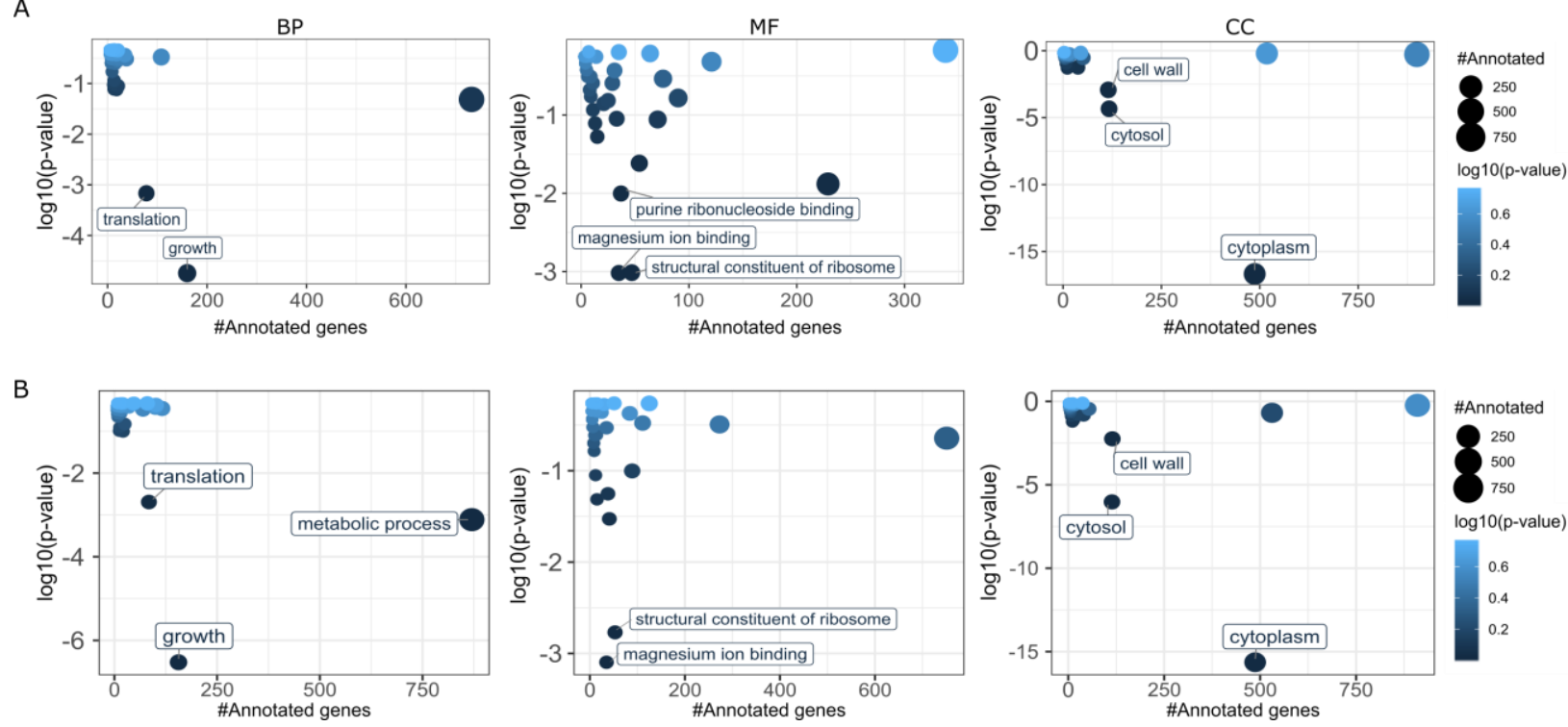

Fig 4. GO enrichment analysis of proteins included in the spectral libraries. GO categories across the three domains biological process (BP), molecular function (MF), and cellular component (CC) were analyzed with a Fisher's exact test $(p$-value $=0.01$ ). The $X$-axis shows the number of annotated genes in each GO category, and the $Y$ axis shows the $\log 10 p$-value. Significantly enriched GO categories ( $p$-value < 0.01 ) in the spectral library are labeled in the figure. The GO category "cytoplasm" had the most significant e-value. (A) Plots for strain EGD-e. (B) Plots for strain ScottA.

The peptides from the two libraries were mapped against the protein-coding sequences of the other genome to identify strain-specific proteotypic peptides and to assess whether some of the strain-specific protein-coding genes were expressed at the protein level. This mapping was done using an in-house tool that extends the original version of PeptideClassifier [59] thereby enabling proteogenomics for prokaryotes [43]. Only $15.5 \%$ and $16.2 \%$ of peptides from EGD-e and ScottA, respectively, were strain-specific proteotypic, indicating that most peptides could be used to quantify protein abundance in both strains and potentially also in other Listeria strains. Additionally, we found that 544 unambiguous peptides of the EGD-e library confirmed expression of 71 of the 191 EGD-e specific protein-coding genes and that 591 Scott-A strain-specific proteotypic peptides in the library confirmed expression of 71 of 269 ScottA-specific proteins (Table 2 and S3 Table). These efforts resulted in a second new proteogenomic resource that enables fast proteotyping of $L$. monocytogenes using a single-tube processing strategy in 
combination with a DIA/SWATH-MS-based workflow that benefits from the generated $L$. monocytogenes spectral libraries. These libraries are now publicly available. Due to the high sensitivity of this spectral library-based DIA/SWATH MS approach in combination with top-end MS instruments, it is now conceivable to gain proteotype information directly from limited numbers of cells extracted from in vivo models of $L$. monocytogenes infection.

Integrated proteogenomic databases as a basis to relate proteotype data back to the genotype

In order to make the generated information accessible for public use, we also created an iPtgxDB for each strain. The concept of iPtgxDBs as a "one-stop shop" for a protein search database that combines the benefits of manual curation efforts with the ability to identify missed smORFs by capturing the entire protein-coding potential of a prokaryotic genome has been described previously [43]. Proteotype data from any condition can be searched against the FASTA file, and experimental evidence (peptides or gene expression data, if available) can be integrated with the GFF file provided, thereby allowing users to visualize experimental evidence for novelties (Fig 1). Alternatively, users may simply compare different annotation resources of a genome sequence or even NCBI RefSeq releases, which can differ substantially.

The minimally redundant iPtgxDBs for L. monocytogenes EGD-e and ScottA strains contained 65,393, and 67,150 proteins, respectively, and were created by integrating and consolidating annotations from RefSeq [42], Prodigal [60], and a modified form of a six-frame translation using the public iPtgxDB web server (https://iptgxdb.expasy.org/iptgxdb/submit/) [43]. Metadata on the number of proteins in each annotation source, the progressive increase of annotation clusters, and the overall number of ORFs in the final iPtgxDB are shown in S5 Table. Proteotype data measured in the control and three GI-mimicking conditions in DDA-MS mode were searched individually against the iPtgxDB fasta file of each strain (see Materials and Methods).

At a stringent peptide-spectrum match (PSM) level FDR $(0.05 \%$, resulting in a protein-level FDR well below 1\%), we obtained unambiguous peptide evidence for 1,907 proteins in EGD-e including 1,899 RefSeq proteins and eight additional novel proteins (S6 Table). The novel proteins 
included two Prodigal-predicted proteins or proteoforms, five in silico ORFs, and one protein with an alternative start site. Furthermore, we observed peptide evidence supporting seven of 28 SNVs in our de novo assembly compared to the EGD-e reference (NC_003210). In ScottA, we observed unambiguous peptide evidence for 1,910 RefSeq proteins including four proteins (3 transposases and 1 cell wall protein) that were missed in the incomplete ScottA reference sequence (Genbank accession: NZ_CM01159; 5 contigs). Additionally, we identified evidence for six novel proteins including three Prodigal proteins, three in silico ORFs, and two alternate protein start sites (S6 Table). Identified novelties include a 45-aa hypothetical protein predicted only by Prodigal that was identified in strain EGD-e by two peptides and 44 PSMs (Fig 5A). Notably, the same 45-aa protein was also identified with these two peptides and 35 PSMs in strain ScottA (data not shown). A BLAST search showed that this SmORF is conserved across L. monocytogenes strains. The second example is the case of a pseudogene predicted by RefSeq in EGD-e (520 amino acids), which contains an internal, C-terminal stop codon. Protein expression evidence was observed for a corresponding smaller Prodigal-predicted protein of 426 amino acids (Fig 5B). Both the Prodigal and Refseq proteins are annotated as formate-tetrahydrofolate ligase. Peptide evidence confirmed expression of the protein to the internal stop codon; this proteoform also contains the P-loop nucleoside triphosphate hydrolase domain that is conserved across $L$. monocytogenes. The third example shows yet another Prodigal prediction for a protein that is 10-aa longer than the corresponding RefSeq protein (which is 212 amino acids) of strain ScottAin (Fig 5C); a peptide supporting the longer proteoform was identified with 26 PSMs. The same peptide was also identified in strain EGD-e with 25 PSMs (data not shown). Finally, one peptide (EVAEELGVHESAVSR, 11 PSMs) supports the non-synonymous amino acid change (caused by an SNV that results in a threonine to alanine codon change) in the protein annotated in our de novo assembly as RNA polymerase factor sigma-54 of 447 amino acids (Fig 5D). S7 Fig shows proteomics evidence (3 peptides, 17 PSMs) for another Prodigal-predicted protein (207 amino acids) annotated as a RpiR family phosphosugar-binding transcriptional regulator; the corresponding Refseq annotation wrongly predicts a pseudogene.

The iPtgxDBs are publicly available (https://iptgxdb.expasy.org/database/). These databases will support efforts in the Listeria community to find proteogenomic evidence for 
bioRxiv preprint doi: https://doi.org/10.1101/668053; this version posted June 13, 2019. The copyright holder for this preprint (which was not certified by peer review) is the author/funder, who has granted bioRxiv a license to display the preprint in perpetuity. It is made available under aCC-BY 4.0 International license.

additional novel smORFs, as pioneered with the example of Prli42 [20]. This type of data has been instrumental in uncovering novelties in several model organisms including the eroded genome of an obligate plant symbiont [61]. Notably, the integration with global dRNA-seq data sets allowed identification of internal start sites of annotated proteins [62], similar to the $\mathrm{N}$ terminomics study in Listeria [20].

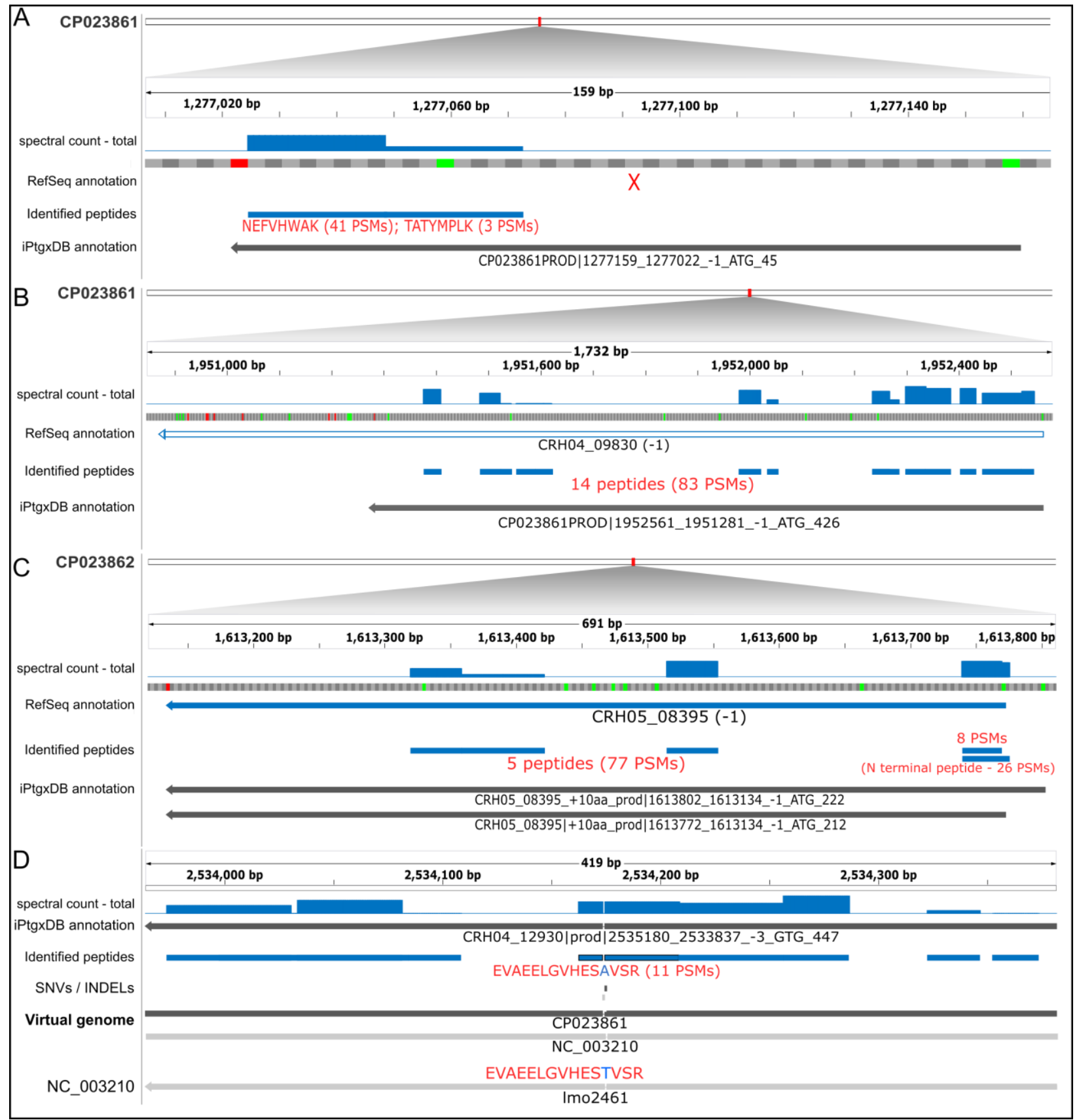


Fig 5. Peptide evidence for novelties identified by proteogenomics. Peptide evidence for (A) a new smORF, (B) an expressed pseudogene, (C) a new start site, and (D) a single amino acid variation (SAAV) uncovered in L. monocytogenes strain EGD-e and/or ScottA. Shown are respective genomic localizations. For $\mathrm{A}-\mathrm{C}$, the respective accession numbers of our assembly are given on the left above various annotation tracks. The iPtgxDB annotation is shown in dark grey, peptide evidence supporting novelties in blue, and a summary of these peptides and PSMs are shown in red. The sequences of the peptides implying the SAAV are shown in panel (D). In the subfigure in panel (D), two genome sequences are compared as a virtual genome, allowing us to overlay experimental evidence. All novelties were discovered by searching data against the strain-specific iPtgxDB. For simplicity, in silico predicted ORFs are not shown.

\section{L. monocytogenes proteotype analysis reveals adaptation during stress}

Overall, approximately 1,700 and 1,900 proteins were identified and quantified (for details see S8 Table) for EGD-e and ScottA, respectively, which represents a major advance for quantitative proteotype profiling studies in Listeria. S9 Table provides a summary over the respective library recovery percentage, data completeness and median CVs for both strains. Average correlation coefficients of biological replicates were above 0.98 indicating a very good reproducibility of sample analysis (data not shown). The reproducibility of the sample analysis was also reflected by the median CVs that ranged around 20\% (S9 Table). Additionally, unsupervised hierarchical clustering revealed clustering of the biological replicates and a distinction across the different conditions tested (data not shown). Notably, the samples incubated with bile salts (duodenum-mimicking condition) demonstrated lower library recovery and a higher number of missing values. This was a result of the significantly lower amount of starting material due to decreased cell viability (Fig 3B). Fig 6 summarizes the proteins found to be differentially 
A
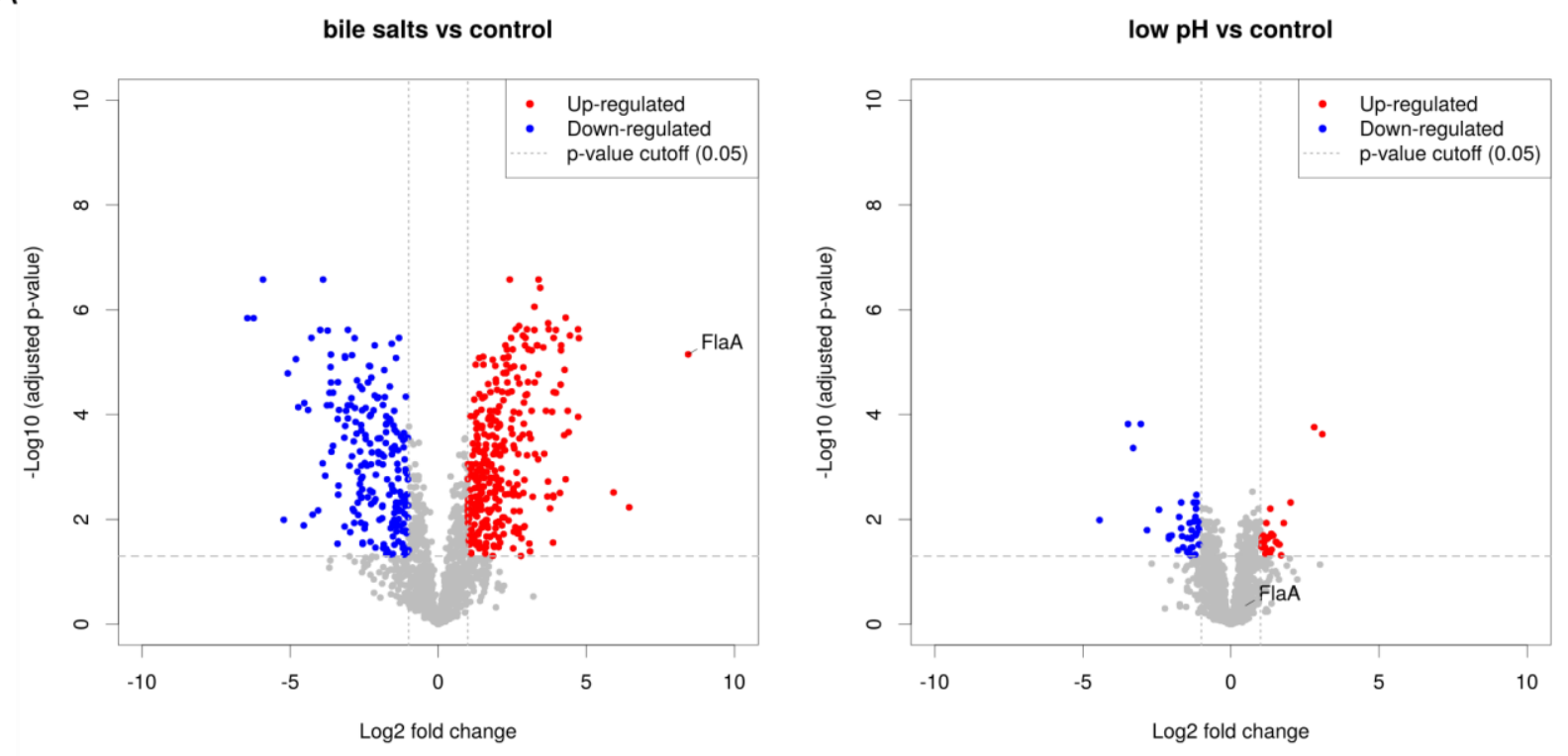

B
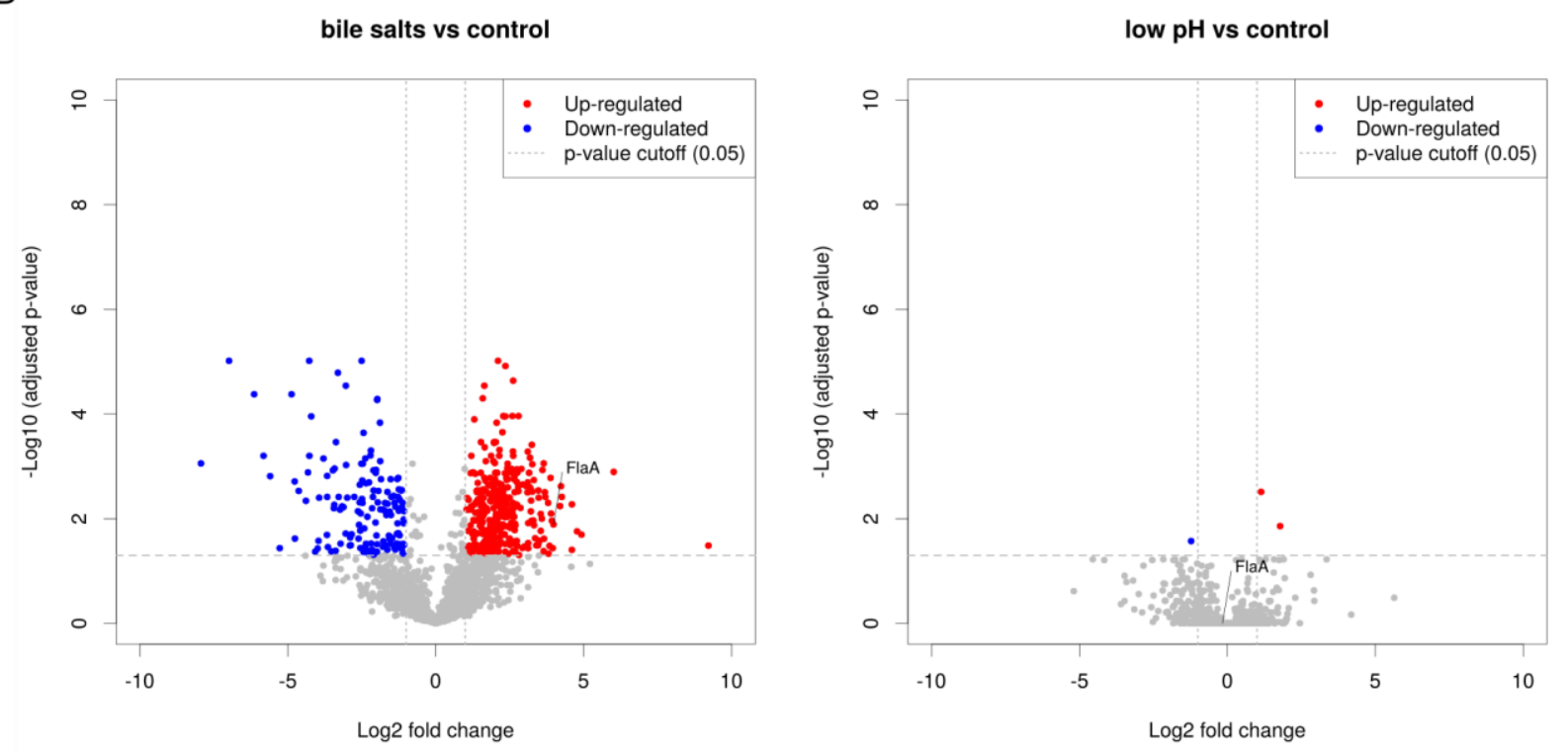

Fig 6. Differential protein abundance in EGD-e and ScottA cells upon exposure to conditions mimicking passage through the upper GI tract. Volcano plots depict the differentially abundant proteins for two conditions, i.e. stomach (low pH) and duodenum (bile salts) compared to control for (A) EGD-e and (B) ScottA. The adjusted p-value (multiple testing corrected, see Material and Methods) is shown as a horizontal gray dashed line, the log2 fold change cut-off of 1 as a vertical dashed gray line. Upregulated proteins are shown in red, downregulated proteins in blue; FlaA is labeled as one example.

abundant in bile salts and low pH compared to the control condition for strains EGD-e (Fig 6A) and ScottA (Fig 6B), respectively. Very few proteins were differentially regulated under high 
osmolarity compared to the control (data not shown). The complete list of differentially regulated proteins under all conditions is included in S10 Table. In general, the condition mimicking those found in the stomach (low $\mathrm{pH} /$ pepsin) resulted in more quantitative changes compared to changes observed under high osmolarity for both strains (S10 Table). Also, more proteins were found to be differentially abundant upon perturbation in EGD-e compared to strain ScottA.

Several studies have investigated the effect of bile salts on L. monocytogenes cells e.g. through gene knockout analyses, and several genes have been associated with resistance to bile (S11 Table). The majority of these proteins is present in our spectral libraries and one of them was specific to the EGD-e genome (CRH04_02340, which corresponds to the product of pva). Interestingly, at the proteotype level, only one of these proteins was found to be significantly differentially abundant when bacterial cells from both strains were incubated with bile salts compared to control condition (bilEB; EDG-e: CRH04_07370, ScottA: CRH05_07950; S11 Table). This likely indicates that the simplistic in vitro conditions developed only partially reflect the full complexity e.g. of the gallbladder fluid, which in other studies had been used from sacrificed animals [63].

One of the most highly differentially regulated proteins in both strains upon bile salt treatment was flagellin, the structural protein of Listeria flagella. This result was unexpected given that flagella are known to be thermoregulated and not formed in temperatures higher than $30{ }^{\circ} \mathrm{C}[64]$. The gene encoding flagellin $(f / a A)$ is located right in between two predicted operons of flagella and motility associated genes ([65]; S11 Table. The majority of these genes was also detected at the protein level and are thus represented in the spectral libraries. For ScottA, the products of three additional flagellar hook-associated genes (flgE, CRH05_04145; flgK, CRH05_04185; flgL, CRH05_04190) were found to be upregulated upon incubation with bile salts. In contrast, in EGD-e, the products of the two component regulatory system (2CRS) cheY (CRH04_3620), a chemotaxis response regulator, and cheA (CRH04_03625) were upregulated in bile and low $\mathrm{pH}$, or bile condition, respectively. Moreover, the list of differentially abundant proteins from all conditions was compared to the list of unique, strain-specific genes to investigate whether strain-specific alterations of the proteotype exist. For EGD-e, nine proteins encoded by the corresponding strain specific genes were differentially abundant across bile (7) 
and low pH (3) conditions with CRH04_001625 (a DUF1433 domain-containing protein) up in both (S10 Table). In contrast, fifteen ScottA strain-specific proteins were differentially regulated upon exposure to bile salts, including three transcriptional regulators (CRH05_01760, CHR05_02245, CRH05_11180; S10 Table). Together, these results suggest that different mechanisms are used by the strains in response to the bile salt stress.

Flagella expression in bile salt condition could hint at a possible escape/survival mechanism

The most striking phenotypic change that was observed in both strains based on the DIA data (Fig 6) was the significant increase of flagellin protein FlaA, the structural protein of flagella, upon exposure to bile salts. The increased abundance of FlaA (Fig 6) was verified independently by parallel reaction monitoring mode (PRM) assays (Fig 7A). Additionally, as revealed by a PRM time-course experiment, protein levels of flagellin had increased after 15 min of incubation with bile salts (Fig 7B). A quantitative real-time PCR experiment was performed to assess flaA mRNA levels. Given that the FlaA protein levels had increased after 15 min of incubation in bilecontaining medium, the qPCR was performed with bacterial cells grown in control and bile conditions for 5, 10, and 15 min. Strikingly, no significant upregulation of flaA mRNA was observed (Fig 7C). This suggests that protein production of FlaA is controlled at the posttranscriptional level . 

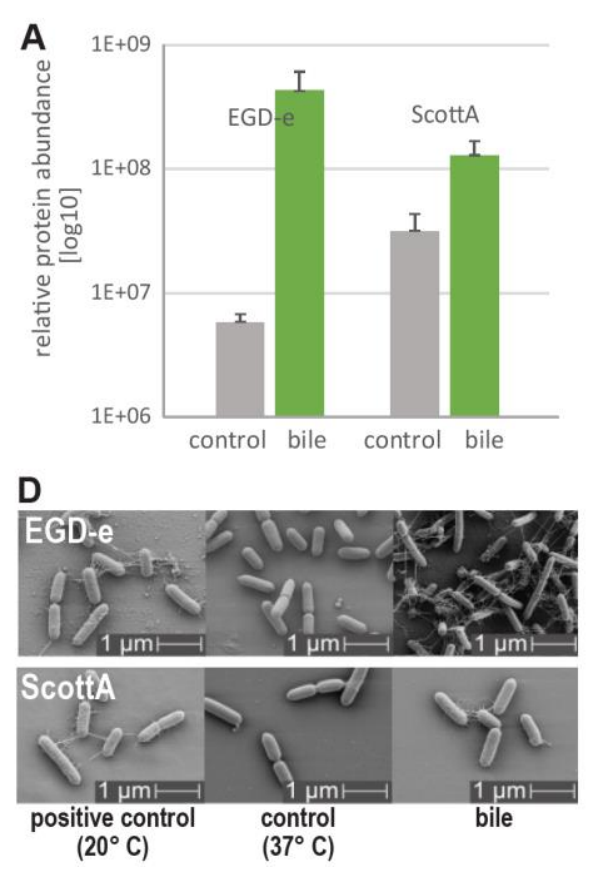

B

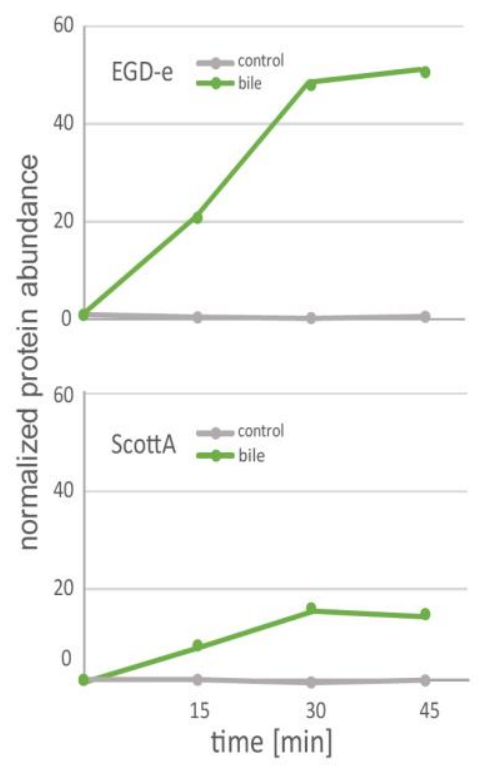

C

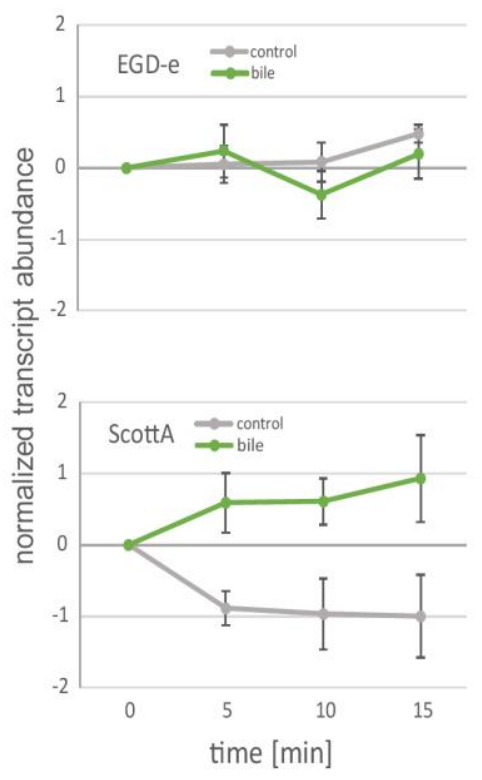

Fig 7. Production of flagella in Listeria strains incubated with bile salts at $37^{\circ} \mathrm{C}$. (A) Barplots of FlaA relative protein abundance based on PRM quantitation. (B) Protein and (C) RNA expression levels of flaA assessed in a time-course experiment using PRM and qPCR, respectively. (D) Representative scanning electron microscopy images of EGD-e and ScottA cells grown in indicated conditions.

To investigate whether the protein increase was the result of the formation of flagella, the cell surfaces of L. monocytogenes cells cultured in control and bile salt containing medium were stained and visualized using light microscopy. Cells grown in the presence of bile salts were flagellated to a larger extent than samples grown in control cultures (data not shown). In order to visualize the flagella at higher resolution, bacterial cells were analyzed by scanning electron microscopy (Fig 7D). The data confirmed that the flagella expression observed at $20^{\circ} \mathrm{C}$ was highly reduced or absent at a temperature of $37^{\circ} \mathrm{C}$. However, upon exposure to bile salts, an increase in flagella expression was readily observed.

The above observations illustrate one valuable example of the additional unique information that can be obtained from quantitative next-gen proteomics but not from gene expression analysis. The presence of flagella under a bile salt stimulus could enable the bacteria to move away from that signal toward the intestinal mucus were they might subsequently lose their flagella and infect the host. Further experiments will be required to elucidate the function 
of flagella in the duodenum. The proteogenomic resources presented here will enable the Listeria community to add comprehensive quantitative proteotype profiling to their functional genomics technology set-up and uncover novel fascinating aspects of Listeria biology (or genotypeproteotype-phenotype relationships), some of which may be regulated at the proteotype level.

\section{Materials and Methods}

\section{Bacterial strains and growth conditions}

Bacterial strain EGD-e (serovar 1/2a) was derived from strain EGD, originally isolated from guinea pigs and used in studies of cell-mediated immunity [19], and differs quite substantially from EGD [65]. ScottA is a clinical strain (serovar 4b) that was isolated during the Massachusetts listeriosis outbreak in 1983 [44]. Cultures were grown to stationary phase in brain heart infusion $(\mathrm{BHI})$ at $30^{\circ} \mathrm{C}$ with shaking and then diluted 1:10 in $\mathrm{BHI}$. Diluted cultures were incubated at $37^{\circ} \mathrm{C}$ until the OD600 was 1. Cells were washed once with PBS and resuspended in the same volume of selected growth medium. Samples were incubated at $37^{\circ} \mathrm{C}$ for $1 \mathrm{~h}$ with shaking, the medium was removed by centrifugation, and cell pellets were frozen until MS analysis. Three media were prepared to resemble conditions encountered in different parts of the upper GI tract [55] with buffered peptone water serving as control medium (BPW: $1 \%(\mathrm{w} / \mathrm{v})$ peptone, $0.5 \%(\mathrm{w} / \mathrm{v}) \mathrm{NaCl}$, $0.35 \%(w / v) \mathrm{Na}_{2} \mathrm{HPO}_{4}, 0.15 \%(\mathrm{w} / \mathrm{v}) \mathrm{KH}_{2} \mathrm{PO}_{4}, \mathrm{pH}$ 7.2). Low pH medium (stomach) was $\mathrm{BPW}, \mathrm{pH} 4$, 1000 units $/ \mathrm{ml}$ pepsin. Bile salts medium (duodenum) was BPW, pH 7, 0.3\% (w/v) bile salts. High osmolarity medium (jejunum) was BPW, pH 8, $0.3 \mathrm{M}$ sucrose. To assess viability, seven serial dilutions of cells were prepared, and $10 \mu \mathrm{l}$ of each sample were spotted on agar plates. Plates were incubated at $30{ }^{\circ} \mathrm{C}$ overnight, and colony counting was performed for the dilution where colonies were well separated from each other. 


\section{Genome sequencing, assembly and annotation}

Genomic DNA was prepared from overnight cultures of EGD-e and ScottA using the Sigma GenElute kit. An insert library was prepared, size selected with BluePippin (fragments $>10 \mathrm{~kb}$ ), and sequenced on the PacBio RSII sequencing platform (1 SMRT cell per strain; P6-C4 chemistry). Initial pre-processing steps (read quality control, pre-assembly) and de novo genome assembly were carried out using HGAP3 [50] as described in detail before [43]. Subsequently, terminal repeats were trimmed, and the contigs were circularized and polished for two rounds using the PacBio pre-assembled reads. The EGD-e chromosome was aligned to the closest NCBI reference (GenBank accession: NC_003210) to adjust the start position according to the reference genome. For ScottA, the circularized contig was start aligned to the $d n a A$ gene as described [53]. Both strains were also sequenced using Illumina MiSeq ( 2 × 300 bp paired end reads); raw fastq reads were mapped to the respective PacBio contigs using BWA-MEM v0.7.12 [66]. The final, highquality genome sequences were submitted to NCBI GenBank and annotated with NCBI's PGAP $4.0[42]$.

\section{Functional annotation}

In addition to the $\mathrm{NCBI}$ annotation, all protein coding genes were also annotated using Interproscan v5.30-69.0 [67] (restricted to hits with an e-value below 1e-5), adding information on Gene Ontology (GO) classification, protein domains, patterns, protein families, profiles, etc. Furthermore, we extracted functional annotations for the protein sequences using eggnogmapper ( $v$ 1.0.3) [68], which transfers functional annotation from orthologous proteins present in EggNOG 4.5 [69]. Prophage sequences were identified and annotated using PHASTER; putative prophages have lower scores than those predicted to be intact [52]. A GO enrichment analysis of the proteins in the spectral library of each strain against all protein-coding genes of the respective strain was performed using the topGO package [70]. A Fisher's exact test with a p-value cut-off of 0.01 was applied to identify significantly enriched GO categories across all three domains (i.e., BC, MF and CC) [71]. 


\section{Comparative genomics}

A comparison of our two de novo assembled, complete genomes was carried out using Roary (v 3.7.0) [54] with standard parameters (minimum identity for blastp set to 50\%, no paralog splitting). From the gene presence/absence output table, we extracted the core and strainspecific protein-coding gene clusters (S3 Table). The unique gene clusters and encoded proteins were subsequently used to identify the subset of proteotypic peptides that allowed quantification of the subset of proteins specific to each strain.

\section{Single-tube sample preparation for DIA/SWATH MS analysis}

A 1-ml culture of approximately $10 \mathrm{e}^{9}$ bacteria was used as a starting material yielding roughly $100 \mu \mathrm{g}$ of total protein. Cell pellets were reconstituted in $500 \mu \mathrm{l}$ of $50 \mathrm{mM}$ ammonium bicarbonate buffer, and $5 \mu \mathrm{g}$ of phage endolysin Ply511 was added. Endolysin was produced in $E$. coli and purified by affinity chromatography as described earlier [72]. The amount and time of endolysin incubation was optimized to allow complete lysis (based on $\mathrm{OD}_{600}$ measurements, data not shown). Samples were incubated under fast end-to-end rotation for $15 \mathrm{~min}$ at $4{ }^{\circ} \mathrm{C}$ and then sonicated at maximum amplitude for $10 \mathrm{sec}$ three times or until viscosity of water was reached. Samples were centrifuged for $10 \mathrm{~min}$ at maximum speed to remove debris, and a BCA protein assay was performed to assess the total protein concentration. For further preparation, $100 \mu \mathrm{g}$ of total protein per sample were used. Proteins were denatured by heat ( $80{ }^{\circ} \mathrm{C}$ for $15 \mathrm{~min}$ ) and addition of $0.1 \%$ acid-cleavable detergent. Proteins were reduced with $10 \mathrm{mM}$ TCEP for $30 \mathrm{~min}$ at $25{ }^{\circ} \mathrm{C}$ and alkylated with $20 \mathrm{mM}$ IAA for $30 \mathrm{~min}$ at $25^{\circ} \mathrm{C}$ in the dark. Proteins were digested with LysC (1:300) for $3 \mathrm{~h}$ at $37^{\circ} \mathrm{C}$ followed by trypsin digestion (1:100) for $16 \mathrm{~h}$ at $37{ }^{\circ} \mathrm{C}$. Upon digestion, samples were acidified with $0.1 \%$ TFA and precipitated detergent was removed by centrifugation. Samples were desalted via mixed cation exchange chromatography and eluted in $1 \mathrm{ml} 5 \% \mathrm{NH}_{4} \mathrm{OH} / 90 \%$ MetOH. Peptides were dried and reconstituted in $60 \mu \mathrm{l}$ 5\% ACN, 0.1\% FA with addition of iRT standard (1:10 v/v). 


\section{DIA/SWATH MS analysis}

Peptides were analysed on an Orbitrap QExactive Plus mass spectrometer (Thermo Scientific) equipped with a nano-electrospray ion source (Thermo Scientific) and coupled to a nano-flow high pressure liquid chromatography (HPLC) pump with an autosampler (EASY-nLC II, Proxeon). Peptides were separated on a reversed-phase chromatography column (75- $\mu \mathrm{m}$ inner diameter PicoTip ${ }^{\text {TM }}$ Emitter, New Objective) that was packed in-house with a C18 stationary phase (Reprosil Gold 120 C18 1.9 mm, Dr. Maisch). Peptides were loaded onto the column with 100\% buffer $\mathrm{A}\left(99.9 \% \mathrm{H}_{2} \mathrm{O}, 0.1 \% \mathrm{FA}\right)$ at 800 bar and eluted at a constant flow rate of $200 \mathrm{nl} / \mathrm{min}$ with a gradient of buffer B (99.9\% ACN, 0.1\% FA) and a subsequent wash step with $90 \%$ buffer B. For the analysis of cell lysates, $3 \mu \mathrm{g}$ of peptides were separated on a $50-\mathrm{cm}$ heated column with a 120-min linear gradient of $5-35 \%$ B, followed by a 10 min gradient to $50 \%$ B and a 5 min gradient to $90 \% \mathrm{~B}$. Between batches of runs, the column was cleaned with two steep consecutive gradients of ACN (10\%-98\%). The MS was operated in DDA mode, with an automatic switch between MS to MS/MS scans. High-resolution MS scans were acquired in the Orbitrap $(120,000$ resolution, automatic gain control target value $2 \times 10^{5}$ ) within a mass range of 395 to $1500 \mathrm{~m} / \mathrm{z}$. The 20 most intense precursor ions (Top20) were fragmented using higher-energy collisional dissociation (HCD) to acquire MS/MS scans in the Orbitrap (30,000 resolution, intensity threshold $2.5 \times 10^{4}$, target value $2 \times 10^{5}$, isolation window $2 \mathrm{~m} / \mathrm{z}$ ). Dynamic exclusion was set to $30 \mathrm{~s}$. Instrument performance was checked by regular quality control measurements using a yeast lysate and the iRT retention time peptide kit (Biognosys), both for DDA and DIA modes.

\section{Database search and spectral library construction}

The RAW files were processed with Proteome Discoverer software, version 2.1 (http://planetorbitrap.com/proteome-discoverer) using the RefSeq protein databases of the de novo assembled EGD-e and ScottA strains with the iRT peptide sequences added. The processing workflow consisted of SequestHT [73] and Amanda [74] search nodes coupled with Percolator [75]. The following search parameters were used for protein identification: (i) peptide mass tolerance set to $10 \mathrm{ppm}$; (ii) MS/MS mass tolerance set to $0.02 \mathrm{Da}$; (iii) fully tryptic peptides with up to two missed cleavages were allowed; (iv) carbamidomethylation of cysteine was set as fixed 
modification, methionine oxidation and protein $\mathrm{N}$-term acetylation were set as variable modifications. Percolator was set at max delta $\mathrm{Cn}$ 0.05, with target FDR strict 0.01 and target FDR relaxed 0.05. The spectral libraries were generated in Spectronaut v10 (Biognosys) using standard parameters including 0.01 peptide spectrum match (PSM) FDR and a Best $\mathrm{N}$-filter with min three and max six fragment ions per peptide.

\section{DIA/SWATH MS sample acquisition and data analysis}

HRM calibration peptides (Biognosys) were spiked into the DIA samples according to the manufacturer's instructions. The samples were analyzed on the same LC-MS system as the DDA runs using identical LC parameters. The mass range m/z 375-1200 was divided into 20 variable windows based on density as described previously [76]. The MS was operated in DIA mode with an automatic switch between MS to MS/MS scans. High-resolution MS scans were acquired in the Orbitrap $\left(35,000\right.$ resolution, automatic gain control target value $\left.5 \times 10^{6}\right)$ within a mass range of 400 to $1220 \mathrm{~m} / \mathrm{z}$. DIA scans preceded an MS1 full scan in the Orbitrap (35,000 resolution, intensity threshold $3 \times 10^{6}$ ) with a stepped NCE 22.5, 25, 27.5. Instrument performance was regularly checked as described above. All DIA data were analyzed directly in Spectronaut v10 (Biognosys) with standard settings (dynamic peak detection, automatic precision nonlinear iRT calibration, interference correction, and cross run normalization (total peak area enabled)). All results were filtered for a q-value of 0.01 (equal to an FDR of $1 \%$ on the peptide level). All other settings were set to default.

\section{Integrated proteogenomics search databases}

IPtgxDBs were created for EGD-e and ScottA by combining the NCBI's RefSeq protein annotation with Prodigal predictions, an $a b$ initio gene predictor [60], and a modified six-frame translation [43]. Proteomics data from the DDA runs were searched against the iPtgxDB FASTA file of each strain individually using MS-GF+ v2017.01.13 [77] to identify evidence for novel ORFs, alternative protein start sites, and SAAVs compared to the reference genome sequence. The search was performed in target-decoy mode, with a precursor mass tolerance of $10 \mathrm{ppm}$, fulltrypticity, maximum precursor charge of 4, carbamidomethylation of cysteine as fixed, and 
methionine oxidation, asparagine deamidation, and protein $\mathrm{N}$-term carbamidomethylation as variable modifications. The search results were filtered for a PSM level FDR of $0.05 \%$, which ensured an estimated protein level FDR below 1\%. In addition, we assessed the proteotypicity of the identified peptides using an in-house version of the original PeptideClassifier [59], further extended to support proteogenomics in prokaryotes [43]. Only peptides that uniquely mapped to one protein (class 1a) were considered for protein identification. Moreover, following an earlier recommendation, we filtered the unambiguous peptides with an additional, variable PSM cut-off [78]: We required two PSMs/peptide for RefSeq annotated proteins; three PSMs per peptide for Prodigal predictions or N-terminal extensions to RefSeq proteins, and four PSMs per peptide for novel in silico predicted proteins. Data were overlaid on top of the GFF file and visualized in a genome browser.

\section{Statistical data evaluation}

All proteomics experiments on EGD-e and ScottA were performed in biological triplicates, except for EGD-e bile, where we could only quantify duplicates. DIA mapping data were searched against the in-house generated spectral libraries using Spectronaut, and the list of quantified spectral features (fragment ions/peptide sequences) was retrieved. In MSstats3 (v3.12.2) [79], which is often used for downstream Spectronaut data processing, the features were logtransformed, and then subjected to median normalization. For feature summarization, the Tukey's median polish algorithm was applied. Protein fold changes and their statistical significance were tested using at least five features per protein. Tests for significant changes in protein abundance across conditions were based on a family of linear mixed-effects models. P values were multiple testing corrected to control the experiment-wide FDR at a desired level using the Benjamini-Hochberg method. Proteins were considered differentially expressed if they showed a fold-change of 2 or higher and an adjusted p-value of 0.05 or lower.

\section{Validation via PRM MS}

Peptides were separated by reversed-phase chromatography on a high-pressure liquid chromatography (HPLC) column (75- $\mu \mathrm{m}$ inner diameter, New Objective) that was packed in- 
house with a 15-cm stationary phase (ReproSil-Pur C18-AQ,1.9 micrometer) and connected to a nano-flow HPLC combined with an autosampler (EASY-nL1000). Peptides were loaded onto the column with $100 \%$ buffer $\mathrm{A}(99.9 \% \mathrm{H} 2 \mathrm{O}, 0.1 \% \mathrm{FA})$ and eluted at a constant flow rate of $300 \mathrm{nl} / \mathrm{min}$ with a 90 min stepped gradient from 3-25\% buffer B (99.9\% ACN, 0.1\% FA) and 25-50\%B. Mass spectra were acquired in PRM on an Orbitrap Fusion ${ }^{\mathrm{TM}}$ Tribrid $^{\mathrm{TM}}$ Mass Spectrometer (Thermo Fisher Scientific). Spectra were acquired at 15,000 resolution (automatic gain control target value $\left.5.0 * 10 \mathrm{e}^{4}\right)$; peptide ions in the mass range of 340-1,400 were monitored. Stepped HCD collision energy was set to $27(+/-5) \%$, maximum injection time to $22 \mathrm{~ms}$. Monitored peptides and results were uploaded to PanoramaWeb (see Data access).

\section{Flagella staining for visualization under light microscopy}

Flagella of L. monocytogenes were stained with Ryu stain using a wet-mount technique. Ryu stain was prepared before every experiment by mixing 1 part solution II with 10 parts solution I. Solution I (mordant) was $10 \mathrm{ml}$ of $5 \%$ aqueous solution of phenol, $2 \mathrm{~g}$ of tannic acid, and $10 \mathrm{ml}$ of saturated aqueous solution of aluminum potassium sulfate-12 hydrate. Solution II (stain) was $12 \mathrm{~g}$ crystal violet in $100 \mathrm{ml}$ of $95 \%$ ethanol. For staining, cells were grown in the desired conditions, and $3 \mu \mathrm{l}$ of culture were transferred on a glass slide and covered with a cover slip leaving small air spaces around the edge. Slides were incubated $10 \mathrm{~min}$ at $25^{\circ} \mathrm{C}$ for bacterial cells to adhere, and $10 \mu \mathrm{l}$ of Ryu stain were applied at the edge of the cover slip. The stain was left to mix with the cell suspension by capillary action. Slides were incubated for $10 \mathrm{~min}$ at $25^{\circ} \mathrm{C}$ and examined under the microscope at 100x (oil).

\section{Flagella visualization using scanning electron microscopy}

Bacteria were cultured as described above, and $0.2 \mathrm{ml}$ of suspension was applied for 20 min on 12-mm coverslips covered with $15 \mathrm{~nm}$ of carbon and coated with poly-L-lysine. After washing twice with PBS, cells were fixed with $2.5 \%$ glutaraldehyde in PBS at room temperature before immediately transferring the samples on ice for $2 \mathrm{~h}$. Samples were then processed in a Pelco Biowave Pro+ tissue processor with use of microwave energy and vacuum. Briefly, the fixed samples underwent a second fixation step in $2.5 \%$ glutaraldehyde, before being washed and 
postfixed in $1 \% \mathrm{OsO}_{4}$ followed by $1 \%$ uranyl acetate in water and dehydration by successive immersion in increasing concentrations of ethanol and finished by critical point drying out of ethanol. The dried coverslips were mounted on SEM aluminium stubs and sputter-coated with 5 $\mathrm{nm}$ of platinum/palladium. SE-images were recorded at $2 \mathrm{kV}$ in a Zeiss Gemini 1530 FEG.

\section{Nucleic acid extraction, purification, and cDNA synthesis}

Bacterial nucleic acids were extracted using a phenol-chloroform protocol adapted from a previous report [80]. Briefly, the bead-beating step was carried out with $500 \mu \mathrm{l}$ of $0.1-\mathrm{mm}$ Zirconia/Silica Beads in a Mixer Mill MM301. Lysis was performed in two rounds of 4 min each with 5 min rest on ice. Nucleic acids were precipitated by addition of 0.1 volume $3 \mathrm{M}$ sodium acetate and 0.6 volume ice-cold isopropanol. The pellet was resuspended in RNase/DNase-free water and subsequently purified with the AllPrep DNA/RNA kit (Qiagen) according to the manufacturer's instructions. DNA isolated from the control culture grown in $\mathrm{BHI}$ at $37^{\circ} \mathrm{C}$ served as positive control. DNA and RNA were quantified with the Qubit ${ }^{\circledR}$ Fluorometer 3.0. RNA was isolated from all samples, and $0.8 \mu \mathrm{g}$ were used for cDNA synthesis (in triplicate) with the $\operatorname{TaqMan}^{\circledR}$ Reverse Transcription Reagents kit according to the manufacturer's instructions using the random hexamer technique. Some reverse-transcription reactions were carried out without enzyme as a control for the absence of DNA in the samples.

qPCR

The reaction was performed in a final volume of $10 \mu \mathrm{l}$ containing $1 X$ SYBR Green Mix, 0.5 $\mu \mathrm{M}$ each of the flaA primers (forward: 5'-GCTGGTCTTGCAGTTGTTACTCGTATG-3'; reverse: 5'CTAATTGACGCATACGTTGCAAGATTG-3') and $1 \mu$ of the diluted DNA template using the SYBRTM Select Master Mix and run on a Rotor-Gene 6000 PCR system with the following program: $50{ }^{\circ} \mathrm{C}$ for $2 \mathrm{~min}, 95^{\circ} \mathrm{C}$ for $2 \mathrm{~min}$, followed by 40 cycles at $95^{\circ} \mathrm{C}$ for $15 \mathrm{~min}$. Three biological replicates were analyzed with samples analyzed in duplicate by qPCR. Raw data were processed with the LinRegPCR program to determine the Cq values, and Python 3.7.0 was used for analysis and to create plots. 


\section{Author contributions}

A.R.V. and M.P.P performed all experiments except those noted below. V.G. performed qPCR.

S.G. performed PRM experiments. A.R.V., M.P.P., S.G. and V.G. analyzed data. Y.S. and M.J.L. supervised V.G. and Listeria experiments in the Loessner laboratory. M.P.P and B.W. conceived the project, C.H.A conceived genomics and proteogenomics aspects. A.R.V, M.P.P, C.H.A. and B.W. designed research. C.H.A., M.P.P, A.R.V and B.W. wrote and revised the manuscript. All authors commented and provided feedback on the manuscript.

\section{Acknowledgements}

The authors thank Ulrich Omasits for bioinformatic support in the early stage of the project and Michael Schmid (both Agroscope) for contributions to the de novo genome assemblies. We would like to thank Patrick Studer (ETH Zurich) who helped with culture of the Listeria strains and J. R. Wyatt for text editing. BW acknowledges support from the Swiss National Science Foundation (SNSF) under grant 31003A_160259, CHA acknowledges support from the SNSF for ARV under grant 31003A-156320.

\section{Data access}

The genome sequences for ScottA and EGD-e are available from NCBI Genbank under accession numbers CP023862 and CP023861, respectively. IPtgxDBs for both strains are available at https://iptgxdb.expasy.org. Targeted MS experiments can be accessed via Panorama (https://panoramaweb.org/660xuF.url). The proteomic data that were used as a basis to develop the spectral libraries and the DIA measurements are available from MASSIVE under ftp://MSV000083881@massive.ucsd.edu (reviewer access login: MSV000083881/ PWD: Listeria_2019). Selected datasets will also be accessible from the Listeriomics web server. 


\section{Figure Legends}

Fig 1. Overview of our next-gen proteogenomics workflow for L. monocytogenes.

Fig 2. Circular plots showing the de novo-assembled genome sequences of EGD-e and ScottA.

Fig 3. Discovery-driven DDA-based Listeria protein expression data obtained under conditions mimicking passage through the upper GI tract.

Fig 4. GO enrichment analysis of proteins included in the spectral libraries.

Fig 5. Peptide evidence for novelties identified by proteogenomics.

Fig 6. Differential protein abundance in EGD-e and ScottA cells upon exposure to conditions mimicking passage through the upper Gl tract.

Fig 7. Production of flagella in Listeria strains incubated with bile salts at $37^{\circ} \mathrm{C}$. 


\section{References}

1. Low JC, Donachie W. A review of Listeria monocytogenes and listeriosis. Vet J. 1997;153: 929.

2. Vázquez-Boland JA, Kuhn M, Berche P, Chakraborty T, Domínguez-Bernal G, Goebel W, et al. Listeria pathogenesis and molecular virulence determinants. Clin Microbiol Rev. 2001;14: 584-640.

3. Hamon M, Bierne H, Cossart P. Listeria monocytogenes: a multifaceted model. Nat Rev Microbiol. 2006;4: 423-434.

4. de Noordhout CM, Devleesschauwer B, Angulo FJ, Verbeke G, Haagsma J, Kirk M, et al. The global burden of listeriosis: a systematic review and meta-analysis. Lancet Infect Dis. 2014;14: 1073-1082.

5. Datta AR, Burall LS. Serotype to genotype: The changing landscape of listeriosis outbreak investigations. Food Microbiol. 2018;75: 18-27.

6. Olier M, Rousseaux S, Piveteau P, Lemaître J-P, Rousset A, Guzzo J. Screening of glutamate decarboxylase activity and bile salt resistance of human asymptomatic carriage, clinical, food, and environmental isolates of Listeria monocytogenes. Int J Food Microbiol. 2004;93: 87-99.

7. Begley M, Gahan CGM, Hill C. The interaction between bacteria and bile. FEMS Microbiol Rev. 2005;29: 625-651.

8. Gahan CGM, Hill C. Listeria monocytogenes: survival and adaptation in the gastrointestinal tract. Front Cell Infect Microbiol. 2014;4: 9.

9. Cossart P. Illuminating the landscape of host-pathogen interactions with the bacterium Listeria monocytogenes. Proc Natl Acad Sci U S A. 2011;108: 19484-19491.

10. Rolhion N, Cossart P. How the study of Listeria monocytogenes has led to new concepts in biology. Future Microbiol. 2017;12: 621-638.

11. Leimeister-Wächter M, Domann E, Chakraborty T. The expression of virulence genes in Listeria monocytogenes is thermoregulated. J Bacteriol. 1992;174: 947-952.

12. Toledo-Arana A, Dussurget O, Nikitas G, Sesto N, Guet-Revillet H, Balestrino D, et al. The Listeria transcriptional landscape from saprophytism to virulence. Nature. 2009;459: 950- 
956.

13. Dabiri GA, Sanger JM, Portnoy DA, Southwick FS. Listeria monocytogenes moves rapidly through the host-cell cytoplasm by inducing directional actin assembly. Proc Natl Acad Sci U S A. 1990;87: 6068-6072.

14. Tilney LG, Portnoy DA. Actin filaments and the growth, movement, and spread of the intracellular bacterial parasite, Listeria monocytogenes. J Cell Biol. 1989;109: 1597-1608.

15. Khan SH, Badovinac VP. Listeria monocytogenes: a model pathogen to study antigen-specific memory CD8 T cell responses. Semin Immunopathol. 2015;37: 301-310.

16. Moura A, Criscuolo A, Pouseele H, Maury MM, Leclercq A, Tarr C, et al. Whole genome-based population biology and epidemiological surveillance of Listeria monocytogenes. Nat Microbiol. 2016;2: 16185.

17. Schmid M, Frei D, Patrignani A, Schlapbach R, Frey JE, Remus-Emsermann MNP, et al. Pushing the limits of de novo genome assembly for complex prokaryotic genomes harboring very long, near identical repeats. Nucleic Acids Res. 2018;46: 8953-8965.

18. Nelson KE, Fouts DE, Mongodin EF, Ravel J, DeBoy RT, Kolonay JF, et al. Whole genome comparisons of serotype $4 b$ and $1 / 2 a$ strains of the food-borne pathogen Listeria monocytogenes reveal new insights into the core genome components of this species. Nucleic Acids Res. 2004;32: 2386-2395.

19. Glaser P, Frangeul L, Buchrieser C, Rusniok C, Amend A, Baquero F, et al. Comparative genomics of Listeria species. Science. 2001;294: 849-852.

20. Impens F, Rolhion N, Radoshevich L, Bécavin C, Duval M, Mellin J, et al. N-terminomics identifies Prli42 as a membrane miniprotein conserved in Firmicutes and critical for stressosome activation in Listeria monocytogenes. Nat Microbiol. 2017;2: 17005.

21. Vogel C, Marcotte EM. Insights into the regulation of protein abundance from proteomic and transcriptomic analyses. Nat Rev Genet. 2012;13: 227-232.

22. Fernández N, Cabrera JJ, Varadarajan AR, Lutz S, Ledermann R, Roschitzki B, et al. An Integrated Systems Approach Unveils New Aspects of Microoxia-Mediated Regulation in Bradyrhizobium diazoefficiens. Frontiers in Microbiology. 2019;10. doi:10.3389/fmicb.2019.00924 
23. Impens F, Radoshevich L, Cossart P, Ribet D. Mapping of SUMO sites and analysis of SUMOylation changes induced by external stimuli. Proc Natl Acad Sci U S A. 2014;111: 1243212437.

24. Kühbacher A, Novy K, Quereda JJ, Sachse M, Moya-Nilges M, Wollscheid B, et al. Listeriolysin O-dependent host surfaceome remodeling modulates Listeria monocytogenes invasion. Pathog Dis. 2018;76. doi:10.1093/femspd/fty082

25. Röst HL, Malmström L, Aebersold R. Reproducible quantitative proteotype data matrices for systems biology. Mol Biol Cell. 2015;26: 3926-3931.

26. Ahrens $\mathrm{CH}$, Brunner E, Qeli E, Basler K, Aebersold R. Generating and navigating proteome maps using mass spectrometry. Nat Rev Mol Cell Biol. 2010;11: 789-801.

27. Omasits U, Quebatte M, Stekhoven DJ, Fortes C, Roschitzki B, Robinson MD, et al. Directed shotgun proteomics guided by saturated RNA-seq identifies a complete expressed prokaryotic proteome. Genome Res. 2013;23: 1916-1927.

28. Schmidt A, Kochanowski K, Vedelaar S, Ahrné E, Volkmer B, Callipo L, et al. The quantitative and condition-dependent Escherichia coli proteome. Nat Biotechnol. 2016;34: 104-110.

29. Aebersold R, Mann M. Mass-spectrometric exploration of proteome structure and function. Nature. 2016;537: 347-355.

30. Bécavin C, Koutero M, Tchitchek N, Cerutti F, Lechat P, Maillet N, et al. Listeriomics: an Interactive Web Platform for Systems Biology of Listeria. mSystems. 2017;2: e00186-16.

31. Misra SK, Moussan Désirée Aké F, Wu Z, Milohanic E, Cao TN, Cossart P, et al. Quantitative proteome analyses identify PrfA-responsive proteins and phosphoproteins in Listeria monocytogenes. J Proteome Res. 2014;13: 6046-6057.

32. Malet JK, Impens F, Carvalho F, Hamon MA, Cossart P, Ribet D. Rapid Remodeling of the Host Epithelial Cell Proteome by the Listeriolysin O (LLO) Pore-forming Toxin. Mol Cell Proteomics. 2018;17: 1627-1636.

33. Melo J, Schrama D, Hussey S, Andrew PW, Faleiro ML. Listeria monocytogenes dairy isolates show a different proteome response to sequential exposure to gastric and intestinal fluids. Int J Food Microbiol. 2013;163: 51-63.

34. He L, Deng Q-L, Chen M-T, Wu Q-P, Lu Y-J. Proteomics analysis of Listeria monocytogenes 
ATCC 19115 in response to simultaneous triple stresses. Arch Microbiol. 2015;197: 833-841.

35. Miyamoto KN, Monteiro KM, da Silva Caumo K, Lorenzatto KR, Ferreira HB, Brandelli A. Comparative proteomic analysis of Listeria monocytogenes ATCC 7644 exposed to a sublethal concentration of nisin. J Proteomics. 2015;119: 230-237.

36. Mata MM, da Silva WP, Wilson R, Lowe E, Bowman JP. Attached and planktonic Listeria monocytogenes global proteomic responses and associated influence of strain genetics and temperature. J Proteome Res. 2015;14: 1161-1173.

37. Aguirre JS, García de Fernando G, Hierro E, Hospital XF, Espinosa I, Fernández M. Characterization of damage on Listeria innocua surviving to pulsed light: Effect on growth, DNA and proteome. Int J Food Microbiol. 2018;284: 63-72.

38. Gillet LC, Navarro P, Tate S, Röst H, Selevsek N, Reiter L, et al. Targeted data extraction of the MS/MS spectra generated by data-independent acquisition: a new concept for consistent and accurate proteome analysis. Mol Cell Proteomics. 2012;11: 0111.016717.

39. Malmström L, Bakochi A, Svensson G, Kilsgård O, Lantz H, Petersson AC, et al. Quantitative proteogenomics of human pathogens using DIA-MS. J Proteomics. 2015;129: 98-107.

40. Schubert OT, Ludwig C, Kogadeeva M, Zimmermann M, Rosenberger G, Gengenbacher M, et al. Absolute Proteome Composition and Dynamics during Dormancy and Resuscitation of Mycobacterium tuberculosis. Cell Host Microbe. 2015;18: 96-108.

41. Alonzo F 3rd, Bobo LD, Skiest DJ, Freitag NE. Evidence for subpopulations of Listeria monocytogenes with enhanced invasion of cardiac cells. J Med Microbiol. 2011;60: 423-434.

42. Tatusova T, DiCuccio M, Badretdin A, Chetvernin V, Nawrocki EP, Zaslavsky L, et al. NCBI prokaryotic genome annotation pipeline. Nucleic Acids Res. 2016;44: 6614-6624.

43. Omasits U, Varadarajan AR, Schmid M, Goetze S, Melidis D, Bourqui M, et al. An integrative strategy to identify the entire protein coding potential of prokaryotic genomes by proteogenomics. Genome Res. 2017;27: 2083-2095.

44. Briers Y, Klumpp J, Schuppler M, Loessner MJ. Genome sequence of Listeria monocytogenes ScottA, a clinical isolate from a food-borne listeriosis outbreak. J Bacteriol. 2011;193: 42844285.

45. Klockgether J, Munder A, Neugebauer J, Davenport CF, Stanke F, Larbig KD, et al. Genome 
diversity of Pseudomonas aeruginosa PAO1 laboratory strains. J Bacteriol. 2010;192: 11131121.

46. Duval $M$, Cossart $P$. Small bacterial and phagic proteins: an updated view on a rapidly moving field. Curr Opin Microbiol. 2017;39: 81-88.

47. Storz G, Wolf YI, Ramamurthi KS. Small Proteins Can No Longer Be Ignored. Annu Rev Biochem. 2014;83: 753-777.

48. Jaffe JD, Berg HC, Church GM. Proteogenomic mapping as a complementary method to perform genome annotation. Proteomics. 2004;4: 59-77.

49. Kyrpides NC, Hugenholtz P, Eisen JA, Woyke T, Göker M, Parker CT, et al. Genomic Encyclopedia of Bacteria and Archaea: Sequencing a Myriad of Type Strains. PLoS Biology. 2014;12: e1001920.

50. Chin C-S, Alexander DH, Marks P, Klammer AA, Drake J, Heiner C, et al. Nonhybrid, finished microbial genome assemblies from long-read SMRT sequencing data. Nat Methods. 2013;10: 563-569.

51. Ross MG, Russ C, Costello M, Hollinger A, Lennon NJ, Hegarty R, et al. Characterizing and measuring bias in sequence data. Genome Biol. 2013;14: R51.

52. Arndt D, Grant JR, Marcu A, Sajed T, Pon A, Liang Y, et al. PHASTER: a better, faster version of the PHAST phage search tool. Nucleic Acids Res. 2016;44: W16-21.

53. Schmid M, Muri J, Melidis D, Varadarajan AR, Somerville V, Wicki A, et al. Comparative Genomics of Completely Sequenced Lactobacillus helveticus Genomes Provides Insights into Strain-Specific Genes and Resolves Metagenomics Data Down to the Strain Level. Front Microbiol. 2018;9: 63.

54. Page AJ, Cummins CA, Hunt M, Wong VK, Reuter S, Holden MTG, et al. Roary: rapid largescale prokaryote pan genome analysis. Bioinformatics. 2015;31: 3691-3693.

55. Barbosa J, Borges S, Magalhães R, Ferreira V, Santos I, Silva J, et al. Behaviour of Listeria monocytogenes isolates through gastro-intestinal tract passage simulation, before and after two sub-lethal stresses. Food Microbiol. 2012;30: 24-28.

56. Loessner MJ, Schneider A, Scherer S. Modified Listeria bacteriophage lysin genes ( $p / y)$ allow efficient overexpression and one-step purification of biochemically active fusion proteins. 
Appl Environ Microbiol. 1996;62: 3057-3060.

57. Savitski MM, Nielsen ML, Zubarev RA. ModifiComb, a new proteomic tool for mapping substoichiometric post-translational modifications, finding novel types of modifications, and fingerprinting complex protein mixtures. Mol Cell Proteomics. 2006;5: 935-948.

58. Donaldson JR, Nanduri B, Burgess SC, Lawrence ML. Comparative Proteomic Analysis of Listeria monocytogenes Strains F2365 and EGD. Appl Environ Microbiol. 2009;75: 366-373.

59. Qeli E, Ahrens CH. PeptideClassifier for protein inference and targeted quantitative proteomics. Nat Biotechnol. 2010;28: 647-650.

60. Hyatt D, Chen G-L, Locascio PF, Land ML, Larimer FW, Hauser L. Prodigal: prokaryotic gene recognition and translation initiation site identification. BMC Bioinformatics. 2010;11: 119.

61. Carlier AL, Omasits U, Ahrens CH, Eberl L. Proteomics analysis of Psychotria leaf nodule symbiosis: improved genome annotation and metabolic predictions. Mol Plant Microbe Interact. 2013;26: 1325-1333.

62. Čuklina J, Hahn J, Imakaev M, Omasits U, Förstner KU, Ljubimov N, et al. Genome-wide transcription start site mapping of Bradyrhizobium japonicum grown free-living or in symbiosis - a rich resource to identify new transcripts, proteins and to study gene regulation. BMC Genomics. 2016;17: 302.

63. Dowd GC, Joyce SA, Hill C, Gahan CGM. Investigation of the mechanisms by which Listeria monocytogenes grows in porcine gallbladder bile. Infect Immun. 2011;79: 369-379.

64. Lemon KP, Higgins DE, Kolter R. Flagellar motility is critical for Listeria monocytogenes biofilm formation. J Bacteriol. 2007;189: 4418-4424.

65. Bécavin C, Bouchier C, Lechat P, Archambaud C, Creno S, Gouin E, et al. Comparison of widely used Listeria monocytogenes strains EGD, 10403S, and EGD-e highlights genomic variations underlying differences in pathogenicity. MBio. 2014;5: e00969-14.

66. Li H. Aligning sequence reads, clone sequences and assembly contigs with BWA-MEM. arXiv. 2013; doi:http://arxiv.org/abs/1303.3997

67. Jones P, Binns D, -Y. Chang H, Fraser M, Li W, McAnulla C, et al. InterProScan 5: genome-scale protein function classification. Bioinformatics. 2014;30: 1236-1240.

68. Huerta-Cepas J, Forslund K, Coelho LP, Szklarczyk D, Jensen U, von Mering C, et al. Fast 
Genome-Wide Functional Annotation through Orthology Assignment by eggNOG-Mapper. Mol Biol Evol. 2017;34: 2115-2122.

69. Huerta-Cepas J, Szklarczyk D, Forslund K, Cook H, Heller D, Walter MC, et al. eggNOG 4.5: a hierarchical orthology framework with improved functional annotations for eukaryotic, prokaryotic and viral sequences. Nucleic Acids Res. 2016;44: D286-93.

70. Alexa A RJ. topGO: Enrichment Analysis for Gene Ontology. R package version 2.34.0. 2018; doi:10.18129/B9.bioc.topGO

71. Alexa A, Rahnenfuhrer J, Lengauer T. Improved scoring of functional groups from gene expression data by decorrelating GO graph structure. Bioinformatics. 2006;22: 1600-1607.

72. Loessner MJ, Wendlinger G, Scherer S. Heterogeneous endolysins in Listeria monocytogenes bacteriophages: a new class of enzymes and evidence for conserved holin genes within the siphoviral lysis cassettes. Mol Microbiol. 1995;16: 1231-1241.

73. Eng JK, McCormack AL, Yates JR. An approach to correlate tandem mass spectral data of peptides with amino acid sequences in a protein database. J Am Soc Mass Spectrom. 1994;5: 976-989.

74. Dorfer V, Pichler P, StranzI T, Stadlmann J, Taus T, Winkler S, et al. MS Amanda, a universal identification algorithm optimized for high accuracy tandem mass spectra. J Proteome Res. 2014;13: 3679-3684.

75. Brosch M, Yu L, Hubbard T, Choudhary J. Accurate and sensitive peptide identification with Mascot Percolator. J Proteome Res. 2009;8: 3176-3181.

76. Bruderer R, Bernhardt OM, Gandhi T, Miladinović SM, Cheng L-Y, Messner S, et al. Extending the limits of quantitative proteome profiling with data-independent acquisition and application to acetaminophen-treated three-dimensional liver microtissues. Mol Cell Proteomics. 2015;14: 1400-1410.

77. Kim S, Pevzner PA. MS-GF+ makes progress towards a universal database search tool for proteomics. Nat Commun. 2014;5: 5277.

78. Nesvizhskii Al. Proteogenomics: concepts, applications and computational strategies. Nat Methods. 2014;11: 1114-1125.

79. Choi M, Chang C-Y, Clough T, Broudy D, Killeen T, MacLean B, et al. MSstats: an R package for 
statistical analysis of quantitative mass spectrometry-based proteomic experiments. Bioinformatics. 2014;30: 2524-2526.

80. Griffiths RI, Whiteley AS, O’Donnell AG, Bailey MJ. Rapid method for coextraction of DNA and RNA from natural environments for analysis of ribosomal DNA- and rRNA-based microbial community composition. Appl Environ Microbiol. 2000;66: 5488-5491. 


\section{Supporting information}

\section{S1 Table. Bacterial strains.}

S2 Table. Overview of genome properties of strains EGD-e and ScottA.

S3 Table. Overview of core genes and genes specific to ScottA and to EGD-e. The subsets of core and strain-specific protein-coding genes are listed for both strains (see separate Excel table). Detailed functional annotation is provided. The 14 genes that were missing in the NCBI reference sequence of ScottA (CM001159.1) are highlighted in orange. Strain-specific genes that are represented in the spectral libraries are marked "TRUE" in the column "present in spectral_library".

S4 Table. Master table. The list of all protein-coding genes in the de novo assembled strains, their functional annotations (NCBI and additional functional annotations; see Materials and Methods), proteomics expression evidence obtained using MS-GF+ after filtering for PSM level FDR of 0.05\% (protein level FDR 1\%), as well as the results of a reciprocal best BLAST hit analyses with the ListiList strain EGD-e (facilitating integration with other datasets) and its corresponding Uniprot accession are shown in a separate Excel file.

S5 Table. Summary of annotation clusters in the iPtgxDBs.

S6 Table. Proteomics evidence for the novelties identified through the proteogenomics search of DDA data for L. monocytogenes strains EGD-e and ScottA. See separate Excel table.

S7 Fig. An incorrectly predicted pseudogene in ScottA. Protein evidence for a 207-amino acid protein ( 3 peptides, 17 PSMs) predicted by Prodigal in L. monocytogenes strain ScottA. It starts from an alternative start codon TTG, which codes for leucine in frame -1; the corresponding longer RefSeq protein (282 amino acids) is wrongly predicted as a pseudogene. Both proteins are annotated as phosphosugar binding transcriptional regulators, and both harbor the RpiR-like SIS (sugar isomerase) protein domains (IPR035472). 
S8 Table: Summary information of precursors, peptides, and protein groups identified for EGDe and ScottA over all conditions using DIA.

S9 Table. Summary of library recovery percentage, data completeness, and median CVs for the DIA dataset.

S10 Table. List of differentially abundant proteins. Proteins identified as differentially abundant in the bile, low $\mathrm{pH}$, and high osmolarity conditions for both strains. Strain-specific genes are highlighted in orange. See separate Excel table.

S11 Table. Candidate genes for bile resistance and operons for flagellar genes. List of Listeria genes previously shown to be involved in bile resistance and genes that are part of two operons with flagellar-related and motility genes that flank the flaA gene (Bécavin C, et al. Comparison of widely used Listeria monocytogenes strains EGD, 10403S, and EGD-e highlights genomic variations underlying differences in pathogenicity. MBio. 2014;5: e00969-14). See separate Excel table. 